\title{
Chiral universality class of normal-superconducting and exciton condensation transitions on surface of topological insulator
}

\author{
Dingping $\mathbf{L i}^{1,2, *}$, Baruch Rosenstein ${ }^{3,4, \dagger}$, B. Ya. Shapiro ${ }^{5, \ddagger}$, I. Shapiro ${ }^{5}$ \\ ${ }^{1}$ School of Physics, Peking University, Beijing 100871, China \\ ${ }^{2}$ Collaborative Innovation Center of Quantum Matter, Beijing 100084, China \\ ${ }^{3}$ Electrophysics Department, National Chiao Tung University, Hsinchu 30050, Taiwan, China \\ ${ }^{4}$ Physics Department, Ariel University, Ariel 40700, Israel \\ ${ }^{5}$ Physics Department, Bar-Ilan University, 52900 Ramat-Gan, Israel \\ Corresponding authors.E-mail: *lidp@pku.edu.cn, ${ }^{\dagger}$ vortexbar@yahoo.com, ${ }^{\ddagger}$ shapib@mail.biu.ac.il \\ Received August 25, 2014; accepted September 14, 2014
}

\begin{abstract}
New two-dimensional systems such as the surfaces of topological insulators (TIs) and graphene offer the possibility of experimentally investigating situations considered exotic just a decade ago. These situations include the quantum phase transition of the chiral type in electronic systems with a relativistic spectrum. Phonon-mediated (conventional) pairing in the Dirac semimetal appearing on the surface of a TI causes a transition into a chiral superconducting state, and exciton condensation in these gapless systems has long been envisioned in the physics of narrow-band semiconductors. Starting from the microscopic Dirac Hamiltonian with local attraction or repulsion, the BardeenCooper-Schrieffer type of Gaussian approximation is developed in the framework of functional integrals. It is shown that owing to an ultrarelativistic dispersion relation, there is a quantum critical point governing the zero-temperature transition to a superconducting state or the exciton condensed state. Quantum transitions having critical exponents differ greatly from conventional ones and belong to the chiral universality class. We discuss the application of these results to recent experiments in which surface superconductivity was found in TIs and estimate the feasibility of phonon pairing.
\end{abstract}

Keywords topological insulator, Weyl semimetal, superconductivity, quantum criticality

PACS numbers 74.20.Fg, 74.90.+n, 74.20.Op

\section{Introduction}

A topological insulator (TI) is a novel state of matter in materials with strong spin-orbit interactions that creates topologically protected surface states $[1-3]$. The electrons (holes) in these states have a linear dispersion relation (see Fig. 1) and can be described approximately by a (pseudo) relativistic two-dimensional (2D) Hamiltonian. The system realizes an ultrarelativistic 2D electron- or hole-conducting liquid, along with the much better studied graphene $[4,5]$, a 2D one-layer sheet of carbon atoms that became a paradigmatic example of a Dirac semimetal. In the context of graphene, certain quantum phase transitions have been theoretically contemplated. Superconductivity in graphene has been repeatedly considered [6-9]; however, despite great experimental efforts, it was never achieved. The $2 \mathrm{D}$ phonons seem to be unable to overcome strong Coulomb repulsion to create a Cooper pair. The same can be said about attempts to achieve exciton condensation in graphene, which was proposed [10-13] even before its discovery. Apparently, the repulsion is not strong enough to create stable electron-hole bound states either [14-17]. The surfaces of TIs therefore became a prime candidate for realizing quantum transitions.

It has long been known that similar $2 \mathrm{D}$ and quasi2D metallic systems like a surface metal on twin planes [18] and layered materials (strongly anisotropic high$T_{c}$ cuprates $[19,20]$ or organic superconductors [21]) may develop 2D (surface) superconductivity. This phenomenon became known as localized superconductivity [22]. Because the best-studied TIs possess a quite standard phonon spectrum [23-25], it was predicted recently [26-30] that they become superconducting. The predicted critical temperature, on the order of $1 \mathrm{~K}$, is rather low, despite a fortunate suppression of the Coulomb repulsion due to a large dielectric constant $\varepsilon \sim 50$. The 
"normal" state (the so-called 2D Weyl semimetal) might yield unusual superconducting properties. The ultrarelativistic nature manifests itself mostly when the Weyl cone is very close to the Fermi surface. Especially interesting is the case in which the chemical potential coincides with the Dirac point (which was originally predicted for the [111] surface of $\mathrm{Bi}_{2} \mathrm{Te}_{3}$ and $\mathrm{Bi}_{2} \mathrm{Se}_{3}$ [31]). Although subsequent angle-resolved photoemission spectroscopy experiments $[1-3]$ showed the cone of surface states to be located on the order of tenths of electron volts away from the Fermi surface, there are experimental means to shift the chemical potential, for example, by applying a bias voltage [32, 33].

Unlike the more customary poor 2D metals with several small pockets of electrons/holes on the Fermi surface (in semiconductor systems or even some high- $T_{c}$ materials $[19,20])$, the electron gas TI has two peculiarities that are especially important when pairing is contemplated. The first is the bipolar nature of the Dirac spectrum: there is no energy gap between the upper and lower cones. The second is that the spin degree of freedom plays a major role in the quasiparticle dynamics. This degree of freedom determines the pairing channel. The pairing channel problem was studied theoretically on the level of the Bogoliubov-de Gennes equation [34]. Both $s$ waves and $p$ waves are possible, and they compete because the bulk inversion symmetry is broken by the surface. Various pairing interactions were considered to calculate the density of states measured in $\mathrm{Cu}_{x} \mathrm{Bi}_{2} \mathrm{Se}_{3}$ using self-consistent analysis [35]. As mentioned above, the most intriguing case is that of a small chemical potential, which has not been addressed microscopically. It has been found to be governed by a quantum critical point (QCP) [36].

The concept of the QCP at zero temperature and various doping levels constitutes a very useful language for describing the microscopic origin of superconductivity in high- $T_{c}$ cuprates and other unconventional superconductors $[19,20]$. Superconducting transitions generally belong to the $U(1)$ class of second-order phase transitions $[37,38]$; however, it was pointed out long ago [39, 40] that, if the normal state dispersion relation is ultrarelativistic, the transition at zero temperature as a function of parameters such as the pairing interaction strength is qualitatively distinct and belongs to the chiral universality classes classified in Refs. [41, 42]. The term "chiral" appears following the corresponding discussion of the chiral-symmetry-breaking transition in quantum chromodynamics, which has been well studied both theoretically and experimentally. Attempts to experimentally identify second-order transitions governed by the QCP in condensed matter included quantum magnets
[36], superconductor-insulator transitions [43], and more recently exciton condensation in graphene $[4,5,44]$ and other Dirac semimetals, including TIs. In the last two cases, the broken symmetry is also often termed "chiral".

Exciton condensation is a very old concept in lowdimensional narrow-gap semiconductor physics. The best-studied exciton condensate is the quantum Hall bilayer at half-filled Landau levels [45-51]. Here, ingenious methods have been developed to separately contact the two layers so that one can directly probe the order parameter via counterflow superfluidity along the layers and tunneling between the layers. The same idea was extended to bilayer graphene and recently to TI [52-55].

In addition, a Dirac semimetal was realized in a cold atom system [56] (following the realization in 2D known as synthetic graphene). Interestingly, the sign and strength of the interaction can be controlled. The Dirac semimetal in optically trapped cold atom systems [56] is well suited to studying this fascinating phenomenon, offering a well-controlled system in which this phenomenon occurs both for repulsive interaction (chiral symmetry breaking) and attractive interaction (superconductivity).

In this paper, the quantum phase transitions in a Dirac semimetal due to local interactions, both attractive (superconductivity) and repulsive (exciton condensation), are studied, with an emphasis on their distinct criticality. The critical exponents belong to chiral universality classes that are identified.

In Section 2, we present the general framework, which makes it possible to study surface superconductivity and exciton condensation in a general Dirac semimetal (a TI, not necessarily time-reversal and reflection invariant, or another of the numerous systems identified recently) with a general local interaction. The local coupling strength $g$, chemical potential $\mu$, and temperature $T$ will be kept general ( $g$ is negative for repulsion, leading to exciton condensation, or positive, leading to superconductivity). Because the symmetry analysis is crucial, we first discuss the space and spin rotations. In Section 3, we concentrate on the simplest time-reversal- and reflection-invariant Dirac model and identify its spontaneous symmetry-breaking patterns. In Section 4, the phase diagram for $g>0$ is obtained for an arbitrary temperature $T$ and a chemical potential much smaller than the Debye energy $T_{D}$. The latter condition is the main difference from the conventional Bardeen-CooperSchrieffer (BCS) model in which $\mu \gg T_{D}$. A QCP appears at $T=\mu=0$ when the coupling strength $g$ reaches a critical value $g_{c}$ depending on the cutoff parameter $T_{D}$. We concentrate on the properties of the superconducting state in a part of the phase diagram that is dominated 
by the QCP. Various critical exponents are obtained. In particular, the coupling strength dependence of the coherence length is $\xi \propto\left(g-g_{c}\right)^{-\nu}$, where $\nu=1$, and the order parameter scales as $\Delta \propto\left(g-g_{c}\right)^{\beta}, \beta=1$. For repulsion, a similar transition occurs in the exciton channel, as described in Section 5. The critical exponents beyond the mean field and the experimental feasibility of superconductivity are discussed in Section 6 .

\section{Generalized mean-field approximation for local four-Fermi interactions}

\subsection{Hamiltonian and partition function}

We consider the second quantized electron Hamiltonian via four-Fermi local coupling of strength $g$ :

$$
\begin{aligned}
& H=\int \mathrm{d}^{2} r \psi_{\alpha}^{\dagger}(\boldsymbol{r}) K_{\alpha \beta} \psi_{\beta}(\boldsymbol{r})-\frac{g}{2} \psi_{\alpha}^{\dagger}(\boldsymbol{r}) \psi_{\beta}^{\dagger}(\boldsymbol{r}) \psi_{\beta}(\boldsymbol{r}) \psi_{\alpha}(\boldsymbol{r}) \\
& K_{\alpha \beta}(\nabla)=\mathcal{E}_{\alpha \beta}(\nabla)-\mu \delta_{\alpha \beta},
\end{aligned}
$$

where the space is two dimensional, $\boldsymbol{r}=\{x, y\}$, and $\mu$ is the chemical potential. The precise definition of the relevant single electronic excitations, $\mathcal{E}_{\alpha \beta}(\nabla)$, will depend on the specific model considered and is specified below. The index $\alpha$ of the spinors $\psi$ refers to the valley/spin degrees of freedom. The partition function is

$$
Z=\operatorname{tre}^{-H / T}=\int \mathrm{D} \psi^{+} \mathrm{D} \psi \mathrm{e}^{-S\left[\psi_{\alpha}^{+}, \psi_{\alpha}\right]},
$$

with a measure defined by the independent Grassmann variables $\mathrm{D} \psi^{+}=\prod_{\alpha} \mathrm{d} \psi_{\alpha}^{+}, \mathrm{D} \psi=\prod_{\alpha} \mathrm{d} \psi_{\alpha}$. The Matsubara action is given by

$$
\begin{aligned}
S\left[\psi^{+}, \psi\right]= & \int_{0}^{1 / T} \mathrm{~d} \tau \int_{r} \psi_{\alpha}^{+}(\tau, \boldsymbol{r})\left(\partial_{\tau}+K_{\alpha \beta}\right) \psi_{\beta}(\tau, \boldsymbol{r}) \\
& -\frac{g}{2} \psi_{\alpha}^{+}(\tau, \boldsymbol{r}) \psi_{\beta}^{+}(\tau, \boldsymbol{r}) \psi_{\beta}(\tau, \boldsymbol{r}) \psi_{\alpha}(\tau, \boldsymbol{r}),
\end{aligned}
$$

with the anti-periodic conditions

$$
\begin{gathered}
\psi_{\alpha}^{+}(\tau, \boldsymbol{r})=-\psi_{\alpha}^{+}(\tau+1 / T, \boldsymbol{r}), \\
\psi_{\alpha}(\tau, \boldsymbol{r})=-\psi_{\alpha}(\tau+1 / T, \boldsymbol{r}) .
\end{gathered}
$$

The local interaction term is not the most general one, but generalization to more exotic local cases (inter-valley [57] exchange spin-spin coupling [58]) is quite straightforward.

The normal and anomalous Green's functions (consistent with the definition in Refs. $[59,60])$ are

$$
\begin{aligned}
& \left\langle T \psi_{\alpha}(X) \psi_{\beta}^{+}\left(X^{\prime}\right)\right\rangle=-G_{\alpha \beta}\left(X ; X^{\prime}\right), \\
& \left\langle T \psi_{\alpha}(X) \psi_{\beta}\left(X^{\prime}\right)\right\rangle=F_{\alpha \beta}\left(X ; X^{\prime}\right), \\
& \left\langle T \psi_{\alpha}^{+}(X) \psi_{\beta}^{+}\left(X^{\prime}\right)\right\rangle=F_{\alpha \beta}^{+}\left(X ; X^{\prime}\right),
\end{aligned}
$$

where $X=(\boldsymbol{r}, \tau)$, and $X^{\prime}=\left(\boldsymbol{r}^{\prime}, \tau^{\prime}\right)$. For simplicity, we denote $\left\langle\psi_{\alpha}(X)\right\rangle$ as $\psi_{\alpha}(X)$ and drop the timeordering operation $T$ in the correlators. For example, $\left\langle\psi_{\alpha}(X) \psi_{\beta}\left(X^{\prime}\right)\right\rangle$ denotes $\left\langle T \psi_{\alpha}(X) \psi_{\beta}\left(X^{\prime}\right)\right\rangle$. Their expressions via a partition function [Eq. (3)] are given in Appendix A.

\subsection{Space and spin rotation symmetries}

In general, a system may be invariant under both space rotation and spin rotation separately. Certain valley symmetries are generally present in Weyl semimetals, and the action is typically invariant only under combined space rotation and spin/valley rotation. The space rotation, $\boldsymbol{r}^{\prime}=\Lambda \boldsymbol{r}$, acts on a generalized spinor field as

$$
\begin{aligned}
& \psi_{\alpha}^{\prime}\left(\boldsymbol{r}^{\prime}, \tau\right)=S(\Lambda)_{\alpha \alpha^{\prime}} \psi_{\alpha^{\prime}}(\boldsymbol{r}, \tau), \\
& \psi_{\beta}^{\prime+}\left(\boldsymbol{r}^{\prime}, \tau\right)=\psi_{\beta^{\prime}}^{+}(\boldsymbol{r}, \tau) S(\Lambda)_{\beta^{\prime} \beta}^{\dagger} .
\end{aligned}
$$

Using the invariance of the action under the transformation, the correlators satisfy

$$
\begin{aligned}
& G_{\alpha \beta}\left(X_{1}, X_{2}\right)=S(\Lambda)_{\alpha \alpha^{\prime}} G_{\alpha^{\prime} \beta^{\prime}}\left(X_{1}, X_{2}\right) S(\Lambda)_{\beta^{\prime} \beta}^{\dagger}, \\
& F_{\alpha \beta}\left(X_{1}, X_{2}\right)=S(\Lambda)_{\alpha \alpha^{\prime}} F_{\alpha^{\prime} \beta^{\prime}}\left(X_{1}, X_{2}\right) S(\Lambda)_{\beta^{\prime} \beta}^{t},
\end{aligned}
$$

or, in matrix form,

$$
\begin{aligned}
& G\left(X_{1}, X_{2}\right)=S(\Lambda) G\left(X_{1}, X_{2}\right) S(\Lambda)^{\dagger}, \\
& F\left(X_{1}, X_{2}\right)=S(\Lambda) F\left(X_{1}, X_{2}\right) S(\Lambda)^{t} .
\end{aligned}
$$

Assuming that the ground state is homogeneous, $G(X, X) \equiv G_{c}$ and $F(X, X) \equiv g^{-1} \Delta$ are constant matrices satisfying

$$
G_{c}=S(\Lambda) G_{c} S(\Lambda)^{+}, \Delta=S(\Lambda) \Delta S(\Lambda)^{t} .
$$

Let $\Sigma$ be the generator of $S(\Lambda)$. Then

$$
\left[\Sigma, G_{c}\right]=0, \quad \Sigma \Delta+\Delta \Sigma^{t}=0 .
$$

$G_{c}$ and $\Delta$ also satisfy the following equations:

$$
G_{c}^{+}=G_{c}, \quad \Delta^{t}=-\Delta .
$$

In a superconductor, $G_{\alpha \beta}^{c}=n_{c} \delta_{\alpha \beta}$, and within the BCS approximation, this results in just renormalization of the chemical potential. Therefore, we finally obtain the Gor'kov equations,

$$
\begin{aligned}
& \left(-\partial_{\tau} \delta_{\alpha \beta}-K_{\alpha \beta}(\nabla)\right) G_{\beta \gamma}\left(X, X^{\prime}\right) \\
& \quad-g F_{\beta \alpha}(X, X) F_{\beta \gamma}^{+}\left(X, X^{\prime}\right)=\delta\left(X-X^{\prime}\right) \delta_{\alpha \gamma}, \\
& \left(\partial_{\tau} \delta_{\alpha \beta}-K_{\beta \alpha}(-\nabla)\right) F_{\beta \gamma}^{+}\left(X, X^{\prime}\right) \\
& \quad-g F_{\alpha \beta}^{+}(X, X) G_{\beta \gamma}\left(X, X^{\prime}\right)=0 .
\end{aligned}
$$

We will also discuss nonsuperconducting states such as an exciton condensate with opposite bulk proper- 
ties, $G_{\alpha \beta}^{c}(X, X) \neq n_{c} \delta_{\alpha \beta}, F_{\alpha \beta}\left(X ; X^{\prime}\right)=0$. In this case, the Dyson-Schwinger form is more convenient. The gap equation can be recast (see Appendix A) in matrix form as

$$
G^{-1}=G_{0}^{-1}+g \delta\left(X-X^{\prime}\right) G_{c}^{\prime},
$$

where $G_{\alpha \beta}^{\prime}$ is the traceless part of $G_{\alpha \beta}$,

$$
\begin{gathered}
G_{\alpha \beta}^{\prime}\left(X ; X^{\prime}\right)=-\left\langle\psi_{\alpha}(X) \psi_{\beta}^{+}\left(X^{\prime}\right)\right\rangle_{c} \\
+\frac{1}{4} \delta_{\alpha \beta} \sum\left\langle\psi_{\gamma}(X) \psi_{\gamma}^{+}\left(X^{\prime}\right)\right\rangle_{c},
\end{gathered}
$$

where the renormalized chemical potential in the equations above.

The Matsubara Green's functions (where $\tau$ is the Matsubara time) for uniform superconducting states can be expressed via Fourier transforms:

$$
\begin{aligned}
& G_{\alpha \beta}\left(\boldsymbol{r}, \tau ; \boldsymbol{r}^{\prime}, \tau^{\prime}\right)=\frac{T}{(2 \pi)^{D}} \int \mathrm{d}^{D} \boldsymbol{k} \\
& \times \mathrm{e}^{\mathrm{i} \boldsymbol{k} \cdot\left(\boldsymbol{r}-\boldsymbol{r}^{\prime}\right)} \sum_{n} \mathrm{e}^{-\mathrm{i} \omega_{n}\left(\tau-\tau^{\prime}\right)} G_{\alpha \beta}\left(\boldsymbol{k}, \omega_{n}\right), \\
& F_{\alpha \beta}^{\dagger}\left(\boldsymbol{r}, \tau ; \boldsymbol{r}^{\prime}, \tau^{\prime}\right)=\frac{T}{(2 \pi)^{D}} \int \mathrm{d}^{D} \boldsymbol{k} \\
& \quad \times \mathrm{e}^{\mathrm{i} \boldsymbol{k} \cdot\left(\boldsymbol{r}-\boldsymbol{r}^{\prime}\right)} \sum_{n} \mathrm{e}^{-\mathrm{i} \omega_{n}\left(\tau-\tau^{\prime}\right)} F_{\alpha \beta}^{\dagger}\left(\boldsymbol{k}, \omega_{n}\right),
\end{aligned}
$$

where $\omega=\pi T(2 n+1)$ is the Matsubara fermionic frequency. Using the Fourier forms of the Matsubara Green's functions can simplify the calculation significantly.

The general formula $[59,60]$ obtained in Appendix B for the energy of the superconducting state is

$$
\frac{\mathrm{d} \Omega}{\mathrm{d}\left(g^{-1}\right)}=\frac{V}{2} \operatorname{tr}\left(\Delta \Delta^{\dagger}\right)
$$

whereas for chiral-symmetry-breaking (exciton condensation) states, the Gaussian energy is [61, 62]

$$
\Omega=-\operatorname{tr}\left\{-\ln G+\left[G_{0}^{-1} G-1\right]\right\}+\frac{g}{2} \operatorname{tr} G_{c} G_{c}^{\dagger} .
$$

\section{Dirac model and its symmetries}

\subsection{Hamiltonian of a time-reversal-invariant TI with} local interaction

Electrons on the surface of a TI perpendicular to the $z$ axis are described by Pauli spinors $\psi(\boldsymbol{r})$, where the upper plane $\boldsymbol{r}=\{x, y\}$. In principle, there are multiple valleys. Cases in which just one valley describes the surface of the TI, such as $\mathrm{Bi}_{2} \mathrm{Te}_{3}$, were considered in Refs. [1-3, $30,34]$. In this case, time-reversal invariance is broken, and chiral symmetry breaking is not allowed, so here we consider the simplest case of multiple valleys: the Dirac model in which the chiralities of the two Weyl modes are opposite, as described by the field operators $\psi_{f s}(\boldsymbol{r})$, where $f=L, R$ are the valley index (pseudospin) for the left/right chirality bands with spin projections taking the values $s=\uparrow, \downarrow$ with respect to, for example, the $z$ axis. To use the Dirac (pseudo-relativistic) notation, these are combined into a four-component bi-spinor creation operator, $\psi^{\dagger}=\left(\psi_{L \uparrow}^{\dagger}, \psi_{L \downarrow}^{\dagger}, \psi_{R \uparrow}^{\dagger}, \psi_{R \downarrow}^{\dagger}\right)$, whose index $\gamma=\{f, s\}$ takes four values. The non-interacting massless Hamiltonian with a Fermi velocity $v_{F}$ and linear dispersion relation (see Fig. 1) is given by $[63,64]$

$$
\mathcal{E}_{\gamma \delta}=-\mathrm{i} \hbar v_{F} \nabla^{i} \alpha_{\gamma \delta}^{i},
$$

where two $4 \times 4$ matrices, $i=x, y$,

$$
\alpha=\left(\begin{array}{cc}
\sigma & 0 \\
0 & -\sigma
\end{array}\right),
$$

are presented in the block form via the Pauli matrices $\boldsymbol{\sigma}$. They are related to the Dirac $\gamma$ matrices (in the chiral representation, this is sometimes called a spinor) by $\boldsymbol{\alpha}=\beta \boldsymbol{\gamma}$, where

$$
\beta=\left(\begin{array}{ll}
0 & 1 \\
1 & 0
\end{array}\right) .
$$

The noninteracting Hamiltonian in this notation is given by

$$
K=\int_{r} \psi^{\dagger}\left(-\mathrm{i} \hbar v_{F} \beta \gamma \cdot \nabla-\mu\right) \psi .
$$

The Matsubara action, Eq. (3), is conveniently written in pseudo-relativistic notation with $\bar{\psi}=\psi^{+} \gamma_{0}$ and (Euclidean) $\gamma_{0}=\mathrm{i} \beta$ :

$$
\begin{aligned}
S= & \int_{\tau=0}^{1 / T} \int_{\boldsymbol{r}}\left[-\bar{\psi}\left(\gamma_{0} \partial_{\tau}+\hbar v_{F} \gamma \cdot \nabla-\mu \gamma_{0}\right) \psi\right. \\
& \left.+\frac{g}{2} \bar{\psi} \gamma_{0} \psi \bar{\psi} \gamma_{0} \psi\right] .
\end{aligned}
$$

Because our focus is on symmetry and its spontaneous breaking, let us review the known discrete and continuous symmetries.

\subsection{Continuous symmetries}

The symmetries of the 2D Dirac model with local interactions [Eqs. (1) and (18)] have been thoroughly discussed in relation to graphene [65]. They include parity, time reversal, and the discrete chiral (flavor) transformation: $\psi \rightarrow \gamma_{5} \psi ; \bar{\psi} \rightarrow-\bar{\psi} \gamma_{5}$, where $\gamma_{5}=\gamma_{0} \gamma_{1} \gamma_{2} \gamma_{3}=\gamma_{5}^{\dagger}$. Spontaneous breaking of this symmetry has been 


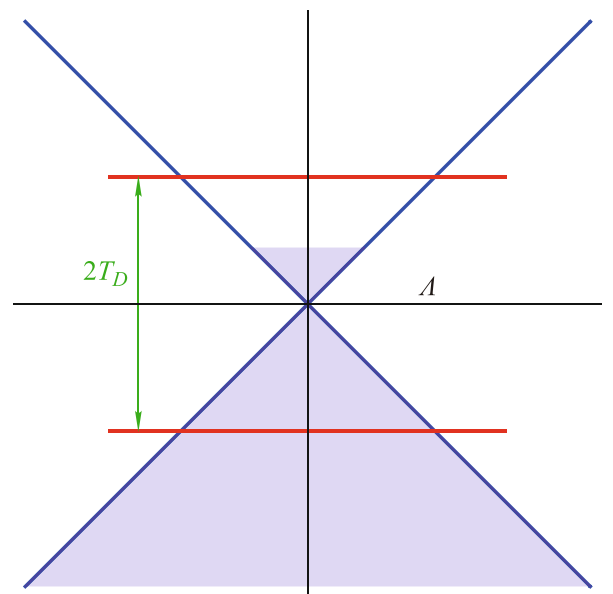

Fig. 1 Schematic picture of band reconstruction of a Weyl semimetal.

comprehensively investigated in the context of graphene [65] and will not be stressed here. There are also three continuous symmetries that in principle can lead to an ordered phase with massless Goldstone bosons (order parameter waves). The first is the usual electric charge $U(1), \psi \rightarrow \mathrm{e}^{\mathrm{i} \chi} \psi$, which is spontaneously broken in a superconducting state studied in the next section. In addition, there are the chiral flavor rotations $S U(2)$, which play an important role in exciton condensation that will be addressed in Section 5 .

\subsubsection{Space-time symmetries: Just Abelian space} rotation combined with spin (pseudospin) rotation

Unlike the non-interacting model, the pseudo-relativistic $2+1$-dimensional Lorentz invariance is explicitly broken by the static interaction in Eq. (1), so only 2D rotations accompanied by the (pseudo) spin rotation already described in Section 2 with the spin $\Sigma$ operator,

$$
\Sigma=\left(\begin{array}{cc}
\sigma_{z} & 0 \\
0 & \sigma_{z}
\end{array}\right)=\gamma_{1} \gamma_{2},
$$

remain symmetric. The conserved quantity is the Abelian angular momentum

$$
J=\int_{\boldsymbol{r}} \psi^{\dagger}(\boldsymbol{r})\left(\mathrm{i} \varepsilon_{i j} r_{i} \nabla_{j}+\frac{1}{2} \boldsymbol{\Sigma}\right) \psi(\boldsymbol{r}) .
$$

The second part is referred to as the spin rotation, $S=\frac{1}{2} \int \psi^{\dagger} \Sigma \psi$.

\subsubsection{Electric charge $U(1)$}

The usual electric charge is $U(1)$ with conserved electric charge:

$$
Q=\int_{\boldsymbol{r}} \psi^{\dagger}(\boldsymbol{r}) \psi(\boldsymbol{r})=\int_{\boldsymbol{r}} \rho(\boldsymbol{r})
$$

The action described by Eq. (22) is invariant under the phase (global gauge) transformation, $\psi \rightarrow \mathrm{e}^{\mathrm{i} \chi} \psi$.

\subsubsection{Flavor (chiral or valley) $S U(2)$}

It was noticed early on in relation to graphene [65] that there is flavor $S U(2)$ symmetry. It is shown in Appendix $\mathrm{C}$ that the quantities

$$
Q_{i}=\int_{\boldsymbol{r}} \psi^{\dagger}(\boldsymbol{r}) T_{i} \psi(\boldsymbol{r})
$$

commute with the Hamiltonian and thus are conserved quantities. The generator matrices,

$$
T_{1}=\frac{\mathrm{i}}{2} \gamma_{3}, \quad T_{2}=\frac{1}{2} \gamma_{5}, \quad T_{3}=\frac{1}{2} \gamma_{3} \gamma_{5},
$$

where $\gamma_{5}=\gamma_{0} \gamma_{1} \gamma_{2} \gamma_{3}$, constitute a nonrelativistic $S U(2)$ algebra:

$$
\left[T_{i}, T_{j}\right]=\mathrm{i} \varepsilon_{i j k} T_{k} .
$$

A discrete chiral symmetry is just chiral rotation by angle $\pi$. The action described by Eq. (22) is invariant under infinitesimal transformation: $\delta \psi=\mathrm{i} T_{i} \psi ; \delta \bar{\psi}=\mathrm{i} \bar{\psi} \gamma_{0} T_{i} \gamma_{0}$. All three continuous symmetries commute (that is, the charge, flavor, and space-time symmetries commute).

\subsection{Spontaneously broken electric charge $U(1)$ symmetry phases: Superconducting pairing channels}

Owing to the locality of the dominant interactions, the superconducting order parameter is local:

$$
O=\int_{\boldsymbol{r}} \psi_{\alpha}^{\dagger}(\boldsymbol{r}) M_{\alpha \beta} \psi_{\beta}^{\dagger}(\boldsymbol{r})
$$

where the constant matrix $M$ should be a $4 \times 4$ antisymmetric matrix. Owing to rotation symmetry, they transform covariantly under infinitesimal rotations generated by the spin $\Sigma$ operator [Eq. (23)].

Out of 16 matrices in the four-dimensional Clifford algebra, 6 are antisymmetric. We will not consider rather exotic phases in which, in addition to the charge $U(1)$ symmetry, neither the $2 \mathrm{D}$ rotations nor the $S U(2)$ chiral transformations are spontaneously broken. Therefore, the superconducting order parameter is invariant under the remaining symmetries: $[O, J]=\left[O, Q_{i}\right]=0$. Namely, it is invariant under $S U(2)$ and is either scalar or pseudoscalar under rotations. The requirement of invariance expressed using Eq. (23) takes the form

$$
[O, J]=\int_{\boldsymbol{r}, \boldsymbol{r}^{\prime}}\left[\psi_{\alpha}^{\dagger}(\boldsymbol{r}) M_{\alpha \beta} \psi_{\beta}^{\dagger}(\boldsymbol{r}) \cdot \psi_{\gamma}^{\dagger}\left(\boldsymbol{r}^{\prime}\right) \boldsymbol{\Sigma}_{\gamma \delta} \psi_{\delta}\left(\boldsymbol{r}^{\prime}\right)\right]
$$




$$
=-\int_{\boldsymbol{r}} \psi^{\dagger}(\boldsymbol{r})\left(\boldsymbol{\Sigma} M+M \boldsymbol{\Sigma}^{t}\right) \psi^{\dagger}(\boldsymbol{r})=0 .
$$

Similarly,

$$
\left[O, Q_{i}\right]=-\int_{\boldsymbol{r}} \psi^{\dagger}(\boldsymbol{r})\left(T_{i} M+M T_{i}^{t}\right) \psi^{\dagger}(\boldsymbol{r})=0 .
$$

One finds that the only scalar is $M=i \alpha_{y}$. There is also a pseudoscalar that will not be discussed here, i.e., we assume that the superconducting state preserves all the other symmetries. Which of the condensates is realized at zero temperature is determined by the parameters of the Hamiltonian along the lines of the dynamical calculation presented in Section 4 for the scalar.

When the effective electron attraction is replaced by repulsion, and superconductivity is not realized, there is still the possibility of continuous symmetry breaking that also belongs to a chiral universality class: the chiral $S U(2)$.

\subsection{Chiral $S U(2)$ broken excitonic phases}

In this section, we consider the opposite situation when the charge symmetry is unbroken, that is, no superconducting condensate appears. Owing to the nontrivial multicomponent situation with the $S U(2)$ symmetry, there are possible transitions into gapped exciton condensate phases. It is plausible that rotational symmetry is also unbroken. However, there are two possible patterns: breakdown to a $U(1)$ subgroup with two Goldstone boson modes, and breakdown to a trivial subgroup with three Goldstone models.

The general order parameter now is

$$
P=\int_{r} \psi_{\alpha}^{\dagger}(\boldsymbol{r}) V_{\alpha \beta} \psi_{\beta}(\boldsymbol{r}),
$$

where the constant matrix $V$ should be a $4 \times 4$ Hermitian matrix. There are four chiral $S U(2)$ triplets of order parameters, $\boldsymbol{P}=\left\{P_{1}, P_{2}, P_{3}\right\}$, which can be viewed as the $O(3)$ vectors. Their commutations with chiral rotation generators $Q_{i}$, as defined in Eq. (26), are

$$
\left[P_{i}, Q_{j}\right]=\int_{\boldsymbol{r}} \psi^{\dagger}(\boldsymbol{r})\left[V_{i}, T_{j}\right] \psi(\boldsymbol{r})=\varepsilon_{i j k} P_{k} .
$$

The four sets of matrices $V_{\alpha \beta}$ in terms of the Dirac and chiral symmetry matrices are

$$
\begin{aligned}
V^{(1)} & =\left\{\gamma_{0} T_{2}, \gamma_{0} T_{1}, \frac{1}{2} \mathrm{i} \gamma_{0}\right\}=\frac{1}{2}\left\{-\gamma_{1} \gamma_{2} \gamma_{3}, \mathrm{i} \gamma_{0} \gamma_{3}, \mathrm{i} \gamma_{0}\right\} \\
V^{(2)} & =\left\{T_{1}, T_{2}, T_{3}\right\}=\frac{1}{2}\left\{\mathrm{i} \gamma_{3}, \gamma_{0} \gamma_{1} \gamma_{2} \gamma_{3},-\gamma_{0} \gamma_{1} \gamma_{2}\right\} \\
V^{(3)} & =\left\{\gamma_{1}, \gamma_{1} \gamma_{3}, \gamma_{0} \gamma_{2} \gamma_{3}\right\} \\
V^{(4)} & =\left\{\gamma_{2}, \gamma_{2} \gamma_{3}, \gamma_{0} \gamma_{1} \gamma_{3}\right\} .
\end{aligned}
$$

There are also four chiral scalars, $\left[P, Q_{i}\right]=0, Q=$ $\int \psi^{\dagger} I \psi$ (charge), $S=\int \psi^{\dagger} \gamma_{1} \gamma_{2} \psi$ (spin), and $H_{i}=$ $\int \psi^{\dagger} \alpha_{i} \psi, \alpha_{i}=\gamma_{0} \gamma_{i}, i=x, y$, which complete the Clifford algebra consisting of 16 Hermitian matrices. These are not order parameters and hence are not of interest to us. We also limit ourselves to the rotation-invariant phases.

The requirement of invariance expressed using Eq. (23) takes the form

$$
[P, J]=\int_{\boldsymbol{r}} \psi^{\dagger}(\boldsymbol{r})[V, \Sigma] \psi^{\dagger}(\boldsymbol{r})
$$

Because $V^{(3)}$ and $V^{(3)}$ in Eq. (34) are not invariant under rotation, only the first two are considered. If only one of the chiral vector order parameters has a nonzero expectation value, say $\left\langle P_{3}^{(1)}\right\rangle \neq 0$, the symmetry breaking pattern is $S U(2) \rightarrow U(1)$, because $\left[P_{3}^{(1)}, Q_{3}\right]=0$. According to the Goldstone theorem, there are two soft modes in the directions $Q_{1}$ and $Q_{2}$. If, in addition, the second vector order parameter reaches the vacuum expectation value, $\left\langle P_{i}^{(2)}\right\rangle \neq 0$, and $i \neq 3$, the pattern will be $S U(2) \rightarrow I$ with three Goldstone modes.

Which symmetry breaking mode is actually realized for a given set of system parameters (e.g., chemical potential, Fermi velocity, interaction sign and strength, temperature) is a dynamical question. Therefore, we now turn to the dynamical aspects of the phase diagram of the Dirac model.

\section{Superconducting state}

Within a Gaussian approximation, the Green's functions obey the Gor'kov equations derived in Ref. [30] and in the last section. For $g>0$, the anomalous Green's functions are nonzero, which simplifies the Gor'kov equations for the Fourier components of the Green's functions considerably:

$$
\begin{aligned}
& D_{\gamma \beta}^{-1} G_{\beta \kappa}(\omega, p)-\widehat{\Delta}_{\gamma \beta} F_{\beta \kappa}^{\dagger}(\omega, p)=\delta^{\gamma \kappa}, \\
& D_{\beta \gamma}^{-1} F_{\beta \kappa}^{\dagger}(\omega, p)+\widehat{\Delta}_{\gamma \beta}^{*} G_{\beta \kappa}(\omega, p)=0,
\end{aligned}
$$

where $D_{\gamma \beta}^{-1}=(\mathrm{i} \omega-\mu) \delta_{\gamma \beta}-v_{F} \varepsilon_{i j} p_{i} \alpha_{\alpha \beta}^{j}$ when the chemical potential is renormalized. The matrix gap function can be chosen as ( $\Delta$ real)

$$
\widehat{\Delta}_{\beta \gamma}=g F_{\gamma \beta}(0)=\left(\begin{array}{cc}
0 & \Delta \\
-\Delta & 0
\end{array}\right) .
$$

These equations are conveniently presented in matrix form (superscript $t$ denotes transposition, and $I$ represents the identity matrix):

$$
D^{-1} G-\widehat{\Delta} F^{\dagger}=I
$$




$$
D^{t-1} F^{\dagger}+\widehat{\Delta}^{*} G=0 .
$$

Solving these equations yields

$$
\begin{aligned}
& G^{-1}=D^{-1}+\widehat{\Delta} D^{t} \widehat{\Delta}^{*}, \\
& F^{\dagger}=-D^{t} \widehat{\Delta}^{*} G,
\end{aligned}
$$

and the gap function is found from the consistency condition

$$
\widehat{\Delta}^{*}=-g \sum_{\omega q} D^{t} \widehat{\Delta}^{*} G
$$

The off-diagonal component of this equation is

$$
\begin{aligned}
\frac{1}{g}= & \sum_{\omega p}\left(\Delta^{2}+v_{F}^{2} p^{2}+\mu^{2}+\hbar^{2} \omega^{2}\right) \\
& \times\left[\Delta^{2}+\hbar^{2} \omega^{2}+\left(v_{F} p-\mu\right)^{2}\right]^{-1} \\
& \times\left[\Delta^{2}+\hbar^{2} \omega^{2}+\left(v_{F} p+\mu\right)^{2}\right]^{-1} .
\end{aligned}
$$

The spectrum of elementary excitations obtained from the poles of the Green's function coincides with that found within the Bogoliubov-de Gennes approach [34]: $E_{p}= \pm \sqrt{\Delta^{2}+\left(v_{F} p-\mu\right)^{2}}$.

\subsection{Zero-temperature phase diagram for the} superconductor-normal transition

At zero temperature, the integrations over frequency and momentum limited by the UV cutoff $\Lambda$ result in

$$
U=\sqrt{\Delta^{2}+\mu^{2}}-\frac{\mu}{2} \log \frac{\sqrt{\Delta^{2}+\mu^{2}}+\mu}{\sqrt{\Delta^{2}+\mu^{2}}-\mu}
$$

where the dependence on the cutoff is incorporated in the renormalized coupling, and the dimension of energy is defined as

$$
U=v_{F} \Lambda-\frac{4 \pi \hbar^{2} v_{F}^{2}}{g} .
$$

This can be interpreted as the effective binding energy of the Cooper pair in the Weyl semimetal. We consider only $\mu>0$, because the particle-hole symmetry makes the opposite case of hole doping, $\mu<0$, identical. Of course, the superconducting solution exists only for $g>0$. In Fig. 2, the dependence of the gap $\Delta$ as a function of the chemical potential $\mu$ is presented for different values of $U$.

For an attractive coupling $g$ stronger than the critical one,

$$
g_{c}=\frac{4 \pi \hbar^{2} v_{F}}{\Lambda} .
$$

When $U>0$ (blue lines in Fig. 2), there are two qualitatively different cases.

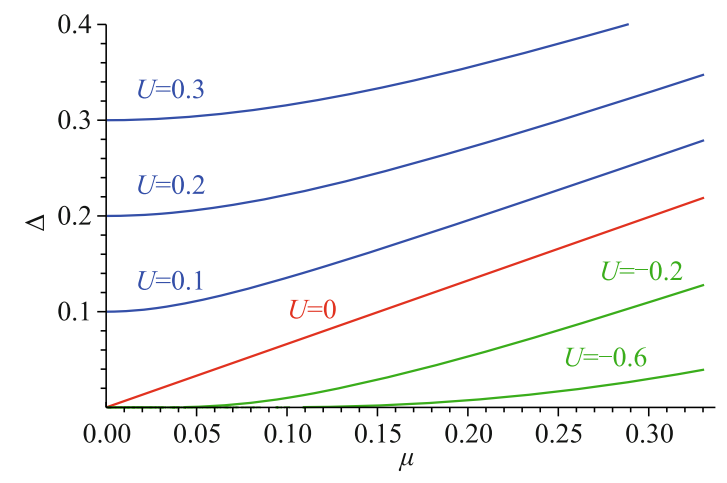

Fig. 2 Order parameter at zero temperature as a function of the chemical potential of the surface TI Weyl semimetal at various coupling values parametrized by the renormalized energy $U$ [Eq. (43)]. For positive $U$ (blue lines), the superconductivity is strong and does not vanish even for zero chemical potential. There exists a critical coupling, $U=0$ (red line), at which the second-order transition occurs at the QCP, $\mu=0$. For negative $U$, superconductivity still exists at $\mu>0$ but is exponentially weak.

(i) When $\mu \ll U$, the dependence of $\Delta$ on the chemical potential is parabolic [30]. In particular, when $\mu=0$, the gap equals $U$. As shown in Fig. 2, the chemical potential has a very limited impact in a large portion of the phase diagram.

(ii) For an attraction just stronger than the critical one, $g>g_{c}$, namely, for small positive $U$, the dependence becomes linear (red line in Fig. $2, \Delta=0.663 \mu$ ). Thus, the already weak condensate becomes sensitive to $\mu$.

Case (i) is more interesting than (ii) because it exhibits stronger superconductivity (larger $T_{c}$; see below). Finally, for $g<g_{c}$, namely, negative $U$ (green lines), the superconductivity is very weak, with an exponential dependence similar to the BCS one, $\Delta \approx \mu$ $\exp [-(|U| / \mu-1)]$. As was mentioned above, in the more interesting cases of large $\Delta$, the dependence on the chemical potential is very weak. A peculiarity of the superconductivity in a TI is that electrons (and holes) in Cooper pairs are themselves created by the pairing interaction rather than being present in the sample as free electrons. Therefore, it is shown that it is possible to neglect the effect of weak doping and consider directly the $\mu=0$ particle-hole symmetric case. This point in parameter space is the QCP [36] and will be studied in detail below. Of course, at finite temperature at any attraction, $g>0$, there exists a (classical) superconducting critical point at a certain temperature $T_{c}$, which is calculated next.

4.2 Dependence of critical temperature $T_{c}$ on the strength of the pairing interaction

Summation over the Matsubara frequency and integra- 


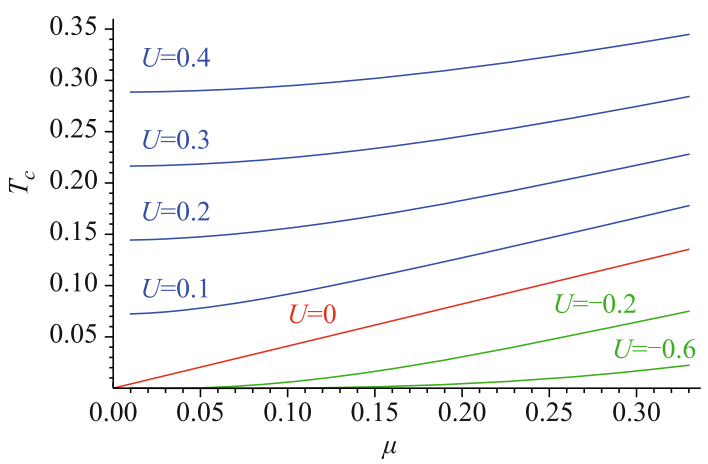

Fig. 3 Transition temperature as a function of the chemical potential at supercritical $(U>0$, blue $)$, critical $(U=0, r e d)$, and subcritical values of coupling.

tions over the momenta in the gap equation, Eq. (42), at finite temperature and arbitrary chemical potential are performed. The critical temperature as a function of $\mu$ and (positive) $U$ is obtained numerically and presented in Fig. 3. Again, at relatively large $U$, the dependence of $T_{c}$ on the chemical potential is very weak and parabolic. When $0<g<g_{c}$, the critical temperature is exponentially small, albeit nonzero, $\frac{\Delta}{U} \approx 1+\left(\frac{\mu}{U}\right)^{2}$.

\subsection{Zero chemical potential $(\mu=0)$}

At zero chemical potential, the Hamiltonian [Eq. (1)] possesses particle-hole symmetry. Microscopically, Cooper pairs of both electrons and holes are formed (see Fig. 1). The system is unique in this sense because the electron-hole symmetry is not spontaneously broken in either the normal or superconducting phase. The supercurrent in such a system does not carry momentum or mass. By obtaining the sum and integrating over the momenta in the gap equation, Eq. (42), analytically [see Appendix A and using the definition of $U$ given in Eq. (43)] for $U>0$, it becomes

$$
U=2 T \log \left(2 \cosh \frac{\Delta}{2 T}\right) .
$$

At zero temperature, $\Delta=U$, whereas $\Delta \rightarrow 0$ as a power of the parameter $U \propto g-g_{c}$ describing the deviation from quantum criticality:

$$
T_{c}=\frac{1}{2 \log 2} U^{z \nu} ; z \nu=1 .
$$

Here $z$ is the dynamical critical exponent [36]. Therefore, as expected, the renormalized coupling describing the deviation from the QCP is proportional to the temperature at which the created condensate disappears.

The temperature dependence of the gap is

$$
\Delta(T)=2 T \operatorname{arccosh}\left(\frac{1}{2} \exp \frac{U}{2 T}\right)
$$

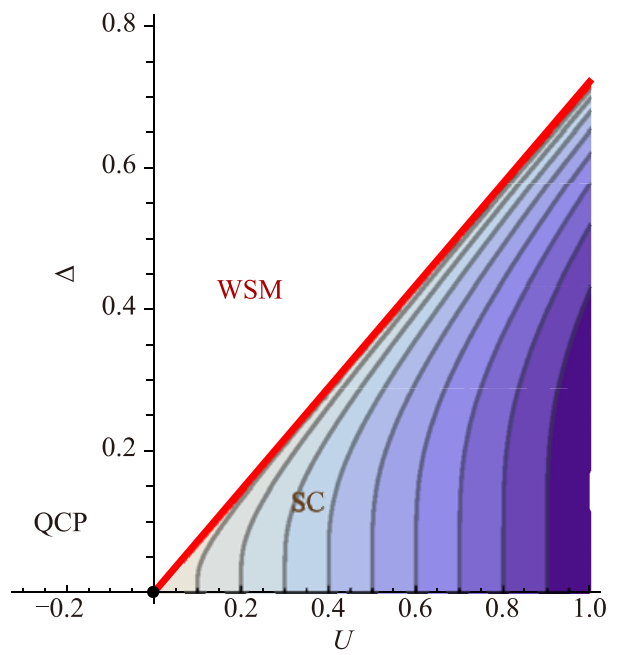

Fig. 4 Phase diagram of surface TI. Order parameter as a function of $U$ describing the deviation from criticality near the QCP at $\Delta=0, \mu=0$. The critical line is a straight line in the mean-field approximation.

This is typical of chiral universality classes [36, 39, 40].

It is interesting to compare this dependence with the conventional BCS $[59,60]$ for transitions at finite temperature, namely, away from the QCP. At zero temperature, $\Delta(0) / T_{c}=2 \log 2=1.39$ (within the BCS model, 1.76), whereas near $T_{c}$, one obtains $\Delta / T_{c}=$ $2^{3 / 2} \log ^{1 / 2} 2 \sqrt{1-t}=2.35 \sqrt{1-t}$ (in the BCS model, $3.07 \sqrt{1-t}$ ), where $t=T / T_{c}$. To describe the behavior of the surface TI in inhomogeneous situations such as an external magnetic field, boundaries, impurities, or junctions with metals or other superconductors, it is necessary to derive the effective theory in terms of the order parameter $\Delta(\boldsymbol{r})$, where $\boldsymbol{r}$ varies on the mesoscopic scale.

A phase diagram of the surface TI order parameter as function of $U$ at zero temperature is plotted in Fig. 4 .

\subsection{Coherence length and condensation energy}

The quadratic term of the Ginzburg-Landau (GL) energy, $F_{2}=\sum_{p} \Delta_{p}^{*} \Gamma(p) \Delta_{p}$, is obtained exactly by expanding the gap equation to linear terms in $\Delta$ for an arbitrary external momentum. The dependence on $\boldsymbol{p}$ is non-analytic, and within our approximation, higher powers of $p$ do not appear. The second term is very different from the quadratic term in the GL functional for conventional phase transitions at finite temperature [37, 38] or even quantum phase transitions in models without Weyl fermions [36], which has a number of qualitative consequences. Comparing the two terms in Eq. (51), one obtains the coherence length as a power of the parameter $U \propto g-g_{c}$ describing the deviation from criticality:

$$
\xi(U)=\frac{\pi}{4} v_{F} \hbar U^{-\nu} ; \quad \nu=1 .
$$


This differs from the dependence in non-chiral universality classes, which is $[37,38] \xi(T) \infty\left(T_{c}-T\right)^{-\nu}, \nu=1 / 2$ in the mean field. Of course, in the regime of critical fluctuations, this exponent is corrected in both non-chiral [37, $38]$ and chiral [41, 42] universality classes.

Local terms in the GL energy density are also exactly calculable. The expression for the kernel can be written as a trace:

$$
\begin{aligned}
\Gamma & =\frac{1}{2} \operatorname{tr}\left(\sum_{\omega q} \sigma^{y} D_{\omega q}^{t} \sigma^{y} D_{\omega, q-p}+\frac{1}{g} I\right) \\
& =\frac{1}{g}-\sum_{\omega q} \frac{\hbar^{2} \omega^{2}-v_{F}^{2} p \cdot q+v_{F}^{2} q^{2}}{\left(\hbar^{2} \omega^{2}+v_{F}^{2} q^{2}\right)\left(\hbar^{2} \omega^{2}+v_{F}^{2}|\boldsymbol{q}-\boldsymbol{p}|^{2}\right)} .
\end{aligned}
$$

Integrating over $\omega$ (at zero temperature) yields

$$
\begin{aligned}
\Gamma= & -\frac{1}{8 \pi^{2} \hbar^{2} v_{F}} \int_{q, \phi} \frac{p q \cos \phi-p^{2}}{|\boldsymbol{q}-\boldsymbol{p}|^{2}+q|\boldsymbol{q}-\boldsymbol{p}|} \\
& -\frac{U}{4 \pi \hbar^{2} v_{F}^{2}},
\end{aligned}
$$

where $\phi$ is the angle between $\boldsymbol{q}$ and $\boldsymbol{p}$. The integral is homogeneous in momentum and therefore is linear in $p=|\boldsymbol{p}|$, and one arrives at

$$
\Gamma(p)=-\frac{U}{4 \pi \hbar^{2} v_{F}^{2}}+\frac{|p|}{16 v_{F} \hbar^{2}} .
$$

4.5 Local terms in Ginzburg-Landau equation and energy

For $p=0$, the gap equation [Eq. (42)] is

$$
\frac{\Delta}{4 \pi \hbar^{2} v_{F}^{2}}\left(-U+\sqrt{\Delta^{*} \Delta}\right)=0 .
$$

This is obtained from the energy functional

$$
F=\frac{1}{4 \pi \hbar^{2} v_{F}^{2}} \int \mathrm{d}^{2} \boldsymbol{r}\left[-U \Delta^{*} \Delta+\frac{2}{3}\left(\Delta^{*} \Delta\right)^{3 / 2}\right] .
$$

It is quite nonstandard compared to the customary quartic term $\left(\Delta^{*} \Delta\right)^{2}$ in conventional universality classes. The GL equations in the homogeneous case for the condensate yield $\Delta_{0}=U^{\beta}$ with a critical exponent $\beta=1$, different from the mean-field value, $\beta=1 / 2$, for the $U(1)$ universality class [37, 38]. The condensation energy density is $f_{0}=-\frac{1}{12 \pi \hbar^{2} v_{F}^{2}} U^{2-\alpha}$, where $\alpha=-1$. The free energy critical exponent at the QCP therefore also differs from the classical $\alpha=0$. The magnetic field is coupled to the order parameter field by a standard minimal substitution. The GL approach is the only available practical tool to study the properties of inhomogeneous configurations [66-68] in an external magnetic field, such as Abrikosov vortex systems.

\section{Exciton condensation}

\subsection{Chiral symmetry breaking}

Assuming that the electric charge $U(1)$ and rotation symmetry are unbroken (no superconductivity) for insufficiently strong attractive interaction (pairing), transitions can still appear owing to the always existing effective repulsion. This possibility has already been considered for graphene [65]. Here we note that the symmetry pattern might be very different from the often-invoked relativistic $2+1$ Gross-Neveu model thoroughly studied as a toy model in relativistic quantum field theory. Possible chiral-symmetry-breaking states were reviewed in Section 2.4, and we consider first a ground state with nonzero order parameter

$$
\left\langle P_{3}^{(1)}\right\rangle=v \neq 0 .
$$

It is convenient to parametrize the Gaussian variational ground state by the trace of the propagator:

$$
G(X ; X)=\frac{m}{g} \gamma_{0},
$$

where the mass $m$ determines the order parameter. According to the gap equation derived in Appendix B, we have

$$
\begin{aligned}
& G^{-1}=(\mathrm{i} \omega+\mu) I-\alpha \cdot\left(\hbar v_{F} k\right)-m \gamma_{0}, \\
& G=-\frac{(\mu+\mathrm{i} \omega) I+\alpha \cdot\left(\hbar v_{F} k\right)+m \gamma_{0}}{m^{2}+\left(\hbar v_{F} k\right)^{2}-(\mu+\mathrm{i} \omega)^{2}},
\end{aligned}
$$

which leads to

$$
\begin{aligned}
m I & =g G^{\prime}(\tau, \boldsymbol{r} ; \tau, \boldsymbol{r}) \gamma_{0}^{-1} \\
& =-\frac{g}{(2 \pi)^{3}} \int_{\omega, \boldsymbol{k}} \frac{m}{m^{2}+\left(\hbar v_{F} \boldsymbol{k}\right)^{2}-(\mu+\mathrm{i} \omega)^{2}} .
\end{aligned}
$$

Integrating with a momentum cutoff $\Lambda$ yields the following for $m \neq 0$ :

$$
1=-\frac{g}{4 \pi\left(\hbar v_{F}\right)^{2}}\left\{\begin{array}{l}
\left(\sqrt{m^{2}+\Lambda^{2}}-m\right), \quad \mu \leqslant m ; \\
\left(\sqrt{m^{2}+\Lambda^{2}}-\mu\right), \quad \mu>m .
\end{array}\right.
$$

For $\mu=m$, one obtains the first-order transition point (for $\mu>0$ ):

$$
\frac{4 \pi\left(\hbar v_{F}\right)^{2}}{\left|g_{c}\right|}=\sqrt{\mu^{2}+\Lambda^{2}}-\mu .
$$

For a larger repulsion, $|g| \geqslant\left|g_{c}\right|$, the mass is larger:

$$
m=\mu+\frac{1}{\left(\hbar v_{F}\right)^{2}}\left(|g|-\left|g_{c}\right|\right)\left(\frac{\Lambda^{2}}{8 \pi}+\frac{2 \pi\left(\hbar v_{F}\right)^{4}}{|g|\left|g_{c}\right|}\right) .
$$

The free energy, according to the Abrikosov formula 
derived in Appendix $\mathrm{B}(\Lambda \gg m)$, is

$$
\begin{array}{r}
{\left[\Omega(m)-\Omega_{0}(m=0)\right] / V=\int_{0}^{m} 2 m^{2} \frac{\mathrm{d} \frac{1}{|g|}}{\mathrm{d} m} \mathrm{~d} m} \\
=-\int_{0}^{m} \frac{m^{2}}{2 \pi\left(\hbar v_{F}\right)^{2}} \mathrm{~d} m=-\frac{m^{3}}{6 \pi\left(\hbar v_{F}\right)^{2}} .
\end{array}
$$

For $\mu>m$, because $\frac{\mathrm{d}(1 /|g|)}{\mathrm{d} m}=\frac{1}{4 \pi\left(\hbar v_{F}\right)^{2}} \frac{m}{\sqrt{m^{2}+\Lambda^{2}}}>0$, the chiral-symmetry-breaking state will have higher energy than the normal one. For $|g|<\left|g_{c}\right|$, the stable ground state is the normal state with $m=0$.

The finite-temperature properties, including the phase diagram and the QCP at zero chemical potential, can be studied along lines similar to those for the superconducting transition.

\section{Discussion and conclusions}

Having considered both the superconducting and excitonic transitions for sufficiently strong attraction or repulsion within the Gaussian approximation, a natural question is what happens when we approach the QCP. At criticality, various renormalization group (RG) methods should be used [37, 38].

\subsection{Criticality beyond the Gaussian approximation}

Critical (quantum) fluctuations are expected to be significant in this relatively low-dimensional (relativistic 2+1dimensional system) system. Generally, they are not as strong as in a 2D statistical system at finite temperature, but they are stronger than in a $3 \mathrm{D}$ one. The approximation we have made describes reasonably well the "Gaussian" fluctuation beyond the region where stronger critical fluctuations in these systems appear and should be treated nonperturbatively [39, 40], typically using variants of the RG approach [37, 38]. The critical exponents in this region differ from the one called "quantum Gaussian (BCS)" in Ref. [36], and the available results were obtained using $\varepsilon$ expansion [41, 42, 69] $(\varepsilon=4-d$, where $d=2+1$ is the space-time dimension); $1 / N$, where $N$ is the number of fermionic species on the surface [41, 42]; or functional (strong coupling) RG [70] and Monte Carlo simulations [71-75] (with reservations specified below). The universality class of the superconducting transition according to the classification proposed in Refs. $[41,42]$ is the chiral XY [symmetry of order parameter $U(1)]$ for $N=1$. The large $N$ expansion is not reliable for the one-component system considered here (however, the number might be larger in similar systems for which our approach trivially generalizes), so let us use the $\varepsilon$ expansion.

According to the formulas for the anomalous dimensions of the order parameter (see Ref. [42]),

$$
\gamma_{\Delta}=\eta=\frac{1}{4} \varepsilon+0.044 \varepsilon^{2}+O\left(\varepsilon^{3}\right) \approx 0.294,
$$

and its square,

$$
\gamma_{\Delta^{2}}=\frac{1+\sqrt{11}}{10} \varepsilon+0.065 \varepsilon^{2} \simeq 0.43 \varepsilon+0.065 \varepsilon^{2},
$$

the critical exponents are obtained from the hyperscaling relations,

$$
\begin{aligned}
& \alpha=2-\frac{d}{2-\gamma_{\Delta^{2}}}=\frac{\varepsilon-2 \gamma_{\Delta^{2}}}{2-\gamma_{\Delta^{2}}}=-0.353, \\
& \beta=\frac{1+\gamma_{\Delta}}{2\left(2-\gamma_{\Delta^{2}}\right)}=0.515
\end{aligned}
$$

and can be compared with those in Table 1 in Ref. [30]. The exponents from the $\varepsilon$ expansion were found to be consistent for larger values of $N$ with the latest Monte Carlo simulations [73-75] and also consistent with the functional RG [70]. The critical exponents of the chiral transition belong to this class with $N=2$.

The corresponding chiral universality class for exciton condensation in Dirac semimetals is the Heisenberg $N=2[S U(2)]$ class. The critical exponents for this case were also calculated in Refs. [41, 42, 69, 70]. They were recently invoked in a discussion of second-order quantum transitions in a Hubbard model on a honeycomb lattice $[76,77]$. Note that the flavor symmetries are often broken explicitly by some type of anisotropy. In this case, Goldstone bosons acquire a small mass (like pions in quantum chromodynamics, in which the chiral symmetry is slightly broken by the light quark masses), although their major properties remain intact. This can be taken into account as a small perturbation [78].

\subsection{Experimental feasibility of observing quantum phase transitions}

The best candidate for the observation of superconductivity is a TI of the $\mathrm{Bi}_{2} \mathrm{Se}_{3}$ family. To estimate the pairing efficiency due to phonons, one should rely on recent studies of surface phonons in TIs [26-29]. The coupling constant in the Hamiltonian [Eq. (1)] is obtained from the exchange of acoustic (Rayleigh) surface phonons, $g=\lambda v_{F}^{2} \hbar^{2} /(2 \pi \mu)$, where $\lambda$ is the dimensionless effective electron-electron interaction constant of order 0.1 (somewhat lower values are obtained in Refs. [79, 80]). It was shown in Refs. [26-29] that at zero temperature, the ratio of $\lambda$ and $\mu$ is constant with a well-defined $\mu \rightarrow 0$ limit, where $g=0.23 \mathrm{eV} \cdot \mathrm{nm}^{2}$ for $v_{F} \approx 7 \times 10^{5} \mathrm{~m} / \mathrm{s}$ (for 
$\mathrm{Bi}_{2} \mathrm{Se}_{3}$ ). The critical coupling constant $g_{c}$ [Eq. (44)] can be estimated from the Debye cutoff $T_{D}=200 \mathrm{~K}$ by determining the momentum cutoff $\Lambda=T_{D} / c_{s}$, where $c_{s}$ is the sound velocity. Taking the value to be $c_{s}=2 \times 10^{3}$ $\mathrm{m} / \mathrm{s}\left(\right.$ for $\mathrm{Bi}_{2} \mathrm{Se}_{3}$ ), one obtains $g_{c}=4 \pi v_{F} c_{s} \hbar^{2} / T_{D}=0.20$ $\mathrm{eV} \mathrm{nm}{ }^{2}$.

Of course, the Coulomb repulsion might weaken or even overpower the effect of the attraction due to phonons, and superconductivity does not occur. In a TI such as $\mathrm{Bi}_{2} \mathrm{Se}_{3}$, however, the dielectric constant is very large $(\varepsilon=50)$, so the Coulomb repulsion is weak. Moreover, it was found in graphene (which has an identical Coulomb interaction) that although the semimetal does not screen [44], the effects of the Coulomb coupling are surprisingly small, even in the leading order in perturbation theory. Superconductivity was observed in these systems, which, however, had to be doped either in the bulk or on the surface [81-84] by supplying $\mathrm{Cu}$ or by applying pressure [85]. It is not yet clear whether the observed superconductivity is a bulk or a surface effect.

The Dirac semimetal in optically trapped cold atoms [56] offers a well-controlled system in which this phenomenon occurs both for the repulsive interaction (chiral symmetry breaking) and in particular for the attractive one (superconductivity) because there is no Coulomb repulsion, as the atoms are neutral.

After the experimental discovery of 3D Dirac semimetals [86-91], a new class of questions similar to those discussed in this paper recently arose. The extraordinary electronic properties of these Dirac materials [92-97], including superconductivity [98] and chiral condensation, are being studied theoretically and experimentally.

\subsection{Conclusions}

We have studied continuous phase transitions in a Dirac semimetal realized recently as a surface TI. The noninteracting system is characterized by a (nearly) zero density of states on the 2D Fermi manifold. It degenerates into a point when the chemical potential coincides with the Weyl point of the surface states, as in the original proposal for a major class of such materials [31]. The pairing attraction (the most plausible candidate being surface phonons) therefore has two roles to play in the creation of a superconducting condensate. The first is to create a pair of electrons (in the present circumstances, that means creating two holes as well), and the second is to pair them. Creating the charges does not cost much energy because the spectrum of the Weyl semimetal is gapless (massless relativistic fermions); this is effective as long as the coupling $g$ is larger than the critical $g_{c}$ [see Eq. (44)]. The situation is more reminiscent of the creation of a chiral condensate in relativistic massless four-fermion theory (a 2D version [39, 40] was recently contemplated for graphene $[4,5,44])$ than of the BCS or even Bose-Einstein condensate in condensed matter systems with a parabolic dispersion law. Owing to the special ultrarelativistic nature of the pairing, transitions at zero temperature as a function of parameters such as the pairing interaction strength are unusual; even the mean-field critical exponents differ from the standard ones, which generally belong to the $U(1)$ class of secondorder phase transitions.

To summarize, we studied the phase diagram of the superconducting and chiral transitions at arbitrary chemical potential, effective local interaction strength, and temperature $T$. The quantum $(T=0)$ critical point appears at zero chemical potential and belongs to the $U_{N}(1)$ chiral universality class (the subscript denotes the number of massless fermions at the QCP according to the classification in Refs. [36, 41, 42]) for attraction (superconductivity) and $S U_{N}(2)$ for repulsion (exciton condensation).

Acknowledgements We are indebted to C. W. Luo, J. J. Lin, and W. B. Jian for explaining the details of their experiments, and T. Maniv and M. Lewkowicz for valuable discussions. The work of D. Li and B. Rosenstein was supported by the NSC of Taiwan, China, Grant No. 98-2112-M-009-014-MY3, and the MOE ATU program. The work of D. Li was also supported by the National Natural Science Foundation of China (Grant No. 11274018). B. Rosenstein was grateful to the School of Physics of Peking University for its hospitality.

Open Access This article is distributed under the terms of the Creative Commons Attribution License which permits any use, distribution, and reproduction in any medium, provided the original author(s) and the source are credited.

\section{Appendix A. Path integral derivation of the Gor'kov and Dyson-Schwinger equations}

\section{A.1 General correlations and sources}

We introduce the Grassmanian source terms into the Matsubara action [Eq. (3)]:

$$
\begin{aligned}
& S\left[\psi^{+}, \psi, J^{+}, J\right]=S\left[\psi^{+}, \psi\right] \\
& -\int_{0}^{\beta} \mathrm{d} \tau \int_{r}\left[\psi_{\sigma}^{+}(\tau, \boldsymbol{r}) J_{\sigma}(\tau, \boldsymbol{r})+\psi_{\sigma}(\tau, \boldsymbol{r}) J_{\sigma}^{+}(\tau, \boldsymbol{r})\right] .
\end{aligned}
$$

The generating functional for the disconnected correlations is $Z\left(J^{+}, J\right)$ :

$$
\left\langle\psi_{\alpha}(X)\right\rangle=\frac{1}{Z} \frac{\delta Z}{\left[-\delta J_{\alpha}^{+}(X)\right]},
$$




$$
\left\langle T \psi_{\alpha}(X) \psi_{\beta}\left(X^{\prime}\right) \ldots\right\rangle_{u}=\frac{1}{Z} \frac{\delta Z}{\left[-\delta J_{\alpha}^{+}(X)\right]\left[-\delta J_{\beta}^{+}\left(X^{\prime}\right)\right] \ldots},
$$

where $X=(\boldsymbol{r}, \tau)$, and $X^{\prime}=\left(\boldsymbol{r}^{\prime}, \tau^{\prime}\right)$. The correlators are (Matsubara) time ordered. The generating functional for the connected correlations is $W=-\ln Z\left(J^{+}, J\right)$ :

$$
\begin{aligned}
& \left\langle\psi_{\alpha}(X)\right\rangle=\frac{\delta W}{\delta J_{\alpha}^{+}(X)}, \\
& \left\langle T \psi_{\alpha}(X) \psi_{\beta}\left(X^{\prime}\right) \ldots\right\rangle=-\frac{\delta W}{\left[-\delta J_{\alpha}^{+}(X)\right]\left[-\delta J_{\beta}^{+}\left(X^{\prime}\right)\right] \ldots} .
\end{aligned}
$$

In particular, we define the normal and anomalous Green's functions (see Ref. [29]) as

$$
\begin{aligned}
\left\langle T \psi_{\alpha}(X) \psi_{\beta}^{+}\left(X^{\prime}\right)\right\rangle & =-\frac{\delta W}{\delta J_{\alpha}(X) \delta J_{\beta}^{+}\left(X^{\prime}\right)} \\
& =-G_{\alpha \beta}\left(X ; X^{\prime}\right), \\
\left\langle T \psi_{\alpha}(X) \psi_{\beta}\left(X^{\prime}\right)\right\rangle & =-\frac{\delta W}{\delta J_{\alpha}^{+}(X) \delta J_{\beta}^{+}\left(X^{\prime}\right)} \\
& =F_{\alpha \beta}\left(X ; X^{\prime}\right), \\
\left\langle T \psi_{\alpha}^{+}(X) \psi_{\beta}^{+}\left(X^{\prime}\right)\right\rangle & =-\frac{\delta W}{\delta J_{\alpha}(X) \delta J_{\beta}\left(X^{\prime}\right)} \\
& =F_{\alpha \beta}^{+}\left(X, X^{\prime}\right),
\end{aligned}
$$

where we denote $\left\langle\psi_{\alpha}(X)\right\rangle$ as $\psi_{\alpha}(X)$, and drop the timeordering operation $T$ in the correlators. For example, $\left\langle\psi_{\alpha}(X) \psi_{\beta}\left(X^{\prime}\right)\right\rangle$ stands for $\left\langle T \psi_{\alpha}(X) \psi_{\beta}\left(X^{\prime}\right)\right\rangle$.

\section{A.2 Derivation of the Gor'kov equation for} superconductivity

When the identities

$$
\begin{aligned}
& \int D \psi^{+} D \psi \frac{\delta}{\delta \psi_{\alpha}^{+}(X)} \mathrm{e}^{-\frac{1}{\hbar} S\left[\psi^{+}, \psi, J^{+}, J\right]}=0 ; \\
& \int D \psi^{+} D \psi \frac{\delta}{\delta \psi_{\alpha}(X)} \mathrm{e}^{-\frac{1}{\hbar} S\left[\psi^{+}, \psi, J^{+}, J\right]}=0,
\end{aligned}
$$

are used, the equations of motion yield

$$
\begin{aligned}
& \left(\hbar \partial_{\tau} \delta_{\alpha \beta}+K_{\alpha \beta}(\nabla)\right) \psi_{\beta}(X) \\
& \quad-g\left\langle\psi_{\beta}^{+}(X) \psi_{\beta}(X) \psi_{\alpha}(X)\right\rangle=J_{\alpha}(\tau, \boldsymbol{r}), \\
& \left(\hbar \partial_{\tau} \delta_{\alpha \beta}-K_{\beta \alpha}(-\nabla)\right) \psi_{\beta}^{+}(X) \\
& \quad+g\left\langle\psi_{\alpha}^{+}(X) \psi_{\beta}^{+}(X) \psi_{\beta}(X)\right\rangle=J_{\alpha}^{+}(\tau, \boldsymbol{r}) .
\end{aligned}
$$

The full correlations of the fields can be generally expressed via connected correlators, for example:

$$
\left\langle\psi_{\beta}^{+}(X) \psi_{\beta}(X) \psi_{\alpha}(X)\right\rangle=\psi_{\beta}^{+}(X) \psi_{\beta}(X) \psi_{\alpha}(X)
$$

$$
\begin{aligned}
& -\psi_{\alpha}(X)\left\langle\psi_{\beta}(X) \psi_{\beta}^{+}(X)\right\rangle_{c} \\
& +\psi_{\beta}(X)\left\langle\psi_{\alpha}(X) \psi_{\beta}^{+}(X)\right\rangle_{c} \\
& +\left\langle\psi_{\beta}^{+}(X)\right\rangle\left\langle\psi_{\beta}(X) \psi_{\alpha}(X)\right\rangle \\
& +\left\langle\psi_{\beta}^{+}(X) \psi_{\beta}(X) \psi_{\alpha}(X)\right\rangle_{c} .
\end{aligned}
$$

For the Gaussian mean-field approximation, we omit higher-order connected correlations, such as $\left\langle\psi_{\beta}^{+}(X) \psi_{\beta}(X) \psi_{\alpha}(X)\right\rangle$ :

$$
\begin{aligned}
& J_{\alpha}(X)=\left(\hbar \partial_{\tau} \delta_{\alpha \beta}+K_{\alpha \beta}\right) \psi_{\beta}(X) \\
& \quad-g \psi_{\beta}^{+}(X) \psi_{\beta}(X) \psi_{\alpha}(X)-\psi_{\alpha}(X)\left\langle\psi_{\beta}(X) \psi_{\beta}^{+}(X)\right\rangle_{c} \\
& \quad+\psi_{\beta}(X)\left\langle\psi_{\alpha}(X) \psi_{\beta}^{+}(X)\right\rangle_{c}+\psi_{\beta}^{+}(X)\left\langle\psi_{\beta}(X) \psi_{\alpha}(X)\right\rangle_{c} .
\end{aligned}
$$

Performing the functional derivative $\frac{\delta}{\delta J_{\gamma}\left(X^{\prime}\right)}$ [by using the identity $\frac{\delta}{\delta J_{\gamma}\left(X^{\prime}\right)} \psi_{\alpha}(X)=\left\langle\psi_{\alpha}(X) \psi_{\gamma}^{+}\left(X^{\prime}\right)\right\rangle$, where the correlators of the odd-numbered Grassmannians vanish) and taking at the end $J_{\alpha}(X)=0$, one obtains:

$$
\begin{aligned}
& \delta\left(X-X^{\prime}\right) \delta_{\alpha \gamma}=\left(\partial_{\tau} \delta_{\alpha \beta}+\widehat{K}_{\alpha \beta}\right)\left\langle\psi_{\beta}(X) \psi_{\gamma}^{+}\left(X^{\prime}\right)\right\rangle \\
& \quad-g\left\{-\left\langle\psi_{\alpha}(X) \psi_{\gamma}^{+}\left(X^{\prime}\right)\right\rangle\left\langle\psi_{\beta}(X) \psi_{\beta}^{+}(X)\right\rangle\right. \\
& \quad+\left\langle\psi_{\beta}(X) \psi_{\gamma}^{+}\left(X^{\prime}\right)\right\rangle\left\langle\psi_{\alpha}(X) \psi_{\beta}^{+}(X)\right\rangle \\
& \left.\quad+\left\langle\psi_{\beta}^{+}(X) \psi_{\gamma}^{+}\left(X^{\prime}\right)\right\rangle\left\langle\psi_{\beta}(X) \psi_{\alpha}(X)\right\rangle\right\},
\end{aligned}
$$

or

$$
\begin{aligned}
& \delta\left(X-X^{\prime}\right) \delta_{\alpha \gamma}=\left(-\partial_{\tau} \delta_{\alpha \beta}-\widehat{K}_{\alpha \beta}\right) G_{\beta \gamma}\left(X, X^{\prime}\right) \\
& \quad-g\left\{-G_{\alpha \gamma}\left(X, X^{\prime}\right) G_{\beta \beta}(X, X)\right. \\
& \quad+G_{\beta \gamma}\left(X, X^{\prime}\right) G_{\alpha \beta}(X, X)_{c} \\
& \left.\quad+F_{\beta \gamma}^{+}\left(X, X^{\prime}\right) F_{\beta \alpha}(X, X)\right\} .
\end{aligned}
$$

Similarly, the second equation of motion,

$$
\begin{aligned}
& J_{\alpha}^{+}(\tau, \boldsymbol{r})=\left(\partial_{\tau} \delta_{\alpha \beta}-\widehat{K}_{\beta \alpha}(-\nabla)\right)\left\langle\psi_{\beta}^{+}(\tau, \boldsymbol{r})\right\rangle \\
& \quad+g\left\{\left\langle\psi_{\alpha}^{+}(\tau, \boldsymbol{r}) \psi_{\beta}^{+}(\tau, \boldsymbol{r})\right\rangle\left\langle\psi_{\beta}(\tau, \boldsymbol{r})\right\rangle\right. \\
& \quad-\left\langle\psi_{\alpha}^{+}(\tau, \boldsymbol{r})\right\rangle\left\langle\psi_{\beta}(\tau, \boldsymbol{r}) \psi_{\beta}^{+}(\tau, \boldsymbol{r})\right\rangle \\
& \left.\quad+\left\langle\psi_{\beta}^{+}(\tau, \boldsymbol{r})\right\rangle\left\langle\psi_{\beta}(\tau, \boldsymbol{r}) \psi_{\alpha}^{+}(\tau, \boldsymbol{r})\right\rangle\right\}
\end{aligned}
$$

yields

$$
\begin{aligned}
& \left(\partial_{\tau} \delta_{\alpha \beta}-\widehat{K}_{\beta \alpha}(-\nabla)\right) F_{\beta \gamma}^{+}\left(X, X^{\prime}\right) \\
& \quad+g\left\{-F_{\alpha \beta}^{+}(X, X) G_{\beta \gamma}\left(X, X^{\prime}\right)\right. \\
& \quad+F_{\alpha \gamma}^{+}\left(X, X^{\prime}\right) G_{\beta \beta}(X, X) \\
& \left.\quad+G_{\beta \alpha}(X, X) F_{\beta \gamma}^{+}\left(X, X^{\prime}\right)\right\}=0 .
\end{aligned}
$$

In the superconducting phase, if the spin-rotation invariance is not broken and chiral symmetry is preserved, 
$G_{\alpha \beta}(X, X)=G_{\alpha \beta}^{c}=n_{c} \delta_{\alpha \beta}$. For the $N$ component spinors $n_{c}=n / N$, where $n$ is the density of electrons, the quadratic parts of Eqs. (74) and (76) simplify to

$$
\begin{aligned}
& -g\left\{-G_{\alpha \gamma}\left(X, X^{\prime}\right) G_{\beta \beta}(X, X)+G_{\beta \gamma}\left(X, X^{\prime}\right) G_{\alpha \beta}(X, X)\right\} \\
& \quad=-g\left\{-G_{\alpha \gamma}\left(X, X^{\prime}\right) N n_{c}+n_{c} G_{\alpha \gamma}\left(X, X^{\prime}\right)\right\} \\
& \quad=g(N-1) n_{c} G_{\alpha \gamma}\left(X, X^{\prime}\right), \\
& g\left\{F_{\alpha \gamma}^{+}\left(X, X^{\prime}\right) G_{\beta \beta}(X, X)+G_{\beta \alpha}(X, X) F_{\beta \gamma}^{+}\left(X, X^{\prime}\right)\right\} \\
& \quad=-g(N-1) n_{c} F_{\alpha \gamma}^{+}\left(X, X^{\prime}\right),
\end{aligned}
$$

and such terms can be absorbed into the chemical potential term, where the chemical potential is replaced by the renormalized

$$
\mu+g(N-1) n_{c}=\mu_{R} .
$$

Therefore, we finally obtain the Gor'kov equations, which are given in Eq. (12).

\section{A.3 Derivation of Dyson-Schwinger equation and renormalized chemical potential for the nonsuperconducting state}

We need only to discuss the equation for $\frac{\delta J_{\alpha}}{\delta \psi_{\beta}}$, as $\frac{\delta J_{\alpha}^{+}}{\delta \psi_{\beta}^{+}}$is just the complex conjugate of $\frac{\delta J_{\alpha}}{\delta \psi_{\beta}}$, and $\frac{\delta J_{\alpha}^{+}}{\delta \psi_{\beta}}=\frac{\delta J_{\alpha}}{\delta \psi_{\beta}^{+}}=0$. We reorganize the equation $\frac{\delta J_{\alpha}}{\delta \psi_{\beta}}$ as

$$
\begin{aligned}
& \frac{\delta J_{\alpha}}{\delta \psi_{\beta}}=\left(\partial_{\tau} \delta_{\alpha \beta}+K_{\alpha \beta}\right) \delta\left(X-X^{\prime}\right)-g \delta\left(X-X^{\prime}\right) \\
& \quad \times\left\{\left\langle\psi_{\alpha}(X) \psi_{\beta}^{+}(X)\right\rangle_{c}-\frac{1}{4} \delta_{\alpha \beta} \sum\left\langle\psi_{\gamma}(X) \psi_{\gamma}^{+}(X)\right\rangle_{c}\right\} \\
& \quad+g \delta_{\alpha \beta} \delta\left(X-X^{\prime}\right)\left\{\left\langle\psi_{\beta}(X) \psi_{\beta}^{+}(X)\right\rangle_{c}\right. \\
& \left.\quad-\sum\left\langle\psi_{\gamma}(X) \psi_{\gamma}^{+}(X)\right\rangle_{c}\right\}
\end{aligned}
$$

The last two terms are proportional to $\delta_{\alpha \beta}$ and can be absorbed into the chemical potential:

$$
\begin{gathered}
\mu+g \delta_{\alpha \beta}\left\{\sum\left\langle\psi_{\gamma}(X) \psi_{\gamma}^{+}(X)\right\rangle_{c}\right. \\
\left.-\left\langle\psi_{\beta}(X) \psi_{\beta}^{+}(X)\right\rangle_{c}\right\}=\mu_{R} .
\end{gathered}
$$

The gap equation can be recast as

$$
\begin{aligned}
& G^{-1}=G_{0}^{-1}+g \delta\left(X-X^{\prime}\right) G^{\prime}\left(X ; X^{\prime}\right), \\
& G_{0}^{-1}=\left(\partial_{\tau} \delta_{\alpha \beta}+K_{\alpha \beta}\right) \delta\left(X-X^{\prime}\right) \\
& G_{\alpha \beta}^{\prime}\left(X ; X^{\prime}\right)=-\left\{\left\langle\psi_{\alpha}(X) \psi_{\beta}^{+}\left(X^{\prime}\right)\right\rangle_{c}\right. \\
& \left.\quad-\frac{1}{4} \delta_{\alpha \beta} \sum\left\langle\psi_{\gamma}(X) \psi_{\gamma}^{+}\left(X^{\prime}\right)\right\rangle_{c}\right\},
\end{aligned}
$$

where $G_{\alpha \beta}^{\prime}$ is the traceless part of $G_{\alpha \beta}$, and the chemical potential in $K_{\alpha \beta}$ is $\mu_{R}$.

\section{Appendix B. Formula for the energy of the superconducting state}

\section{B.1 Gap equation in Nambu notation}

Nambu notation is a compact representation of the Gor'kov equations for superconductors:

$$
\begin{aligned}
& \mathcal{G}_{\alpha \beta}\left(X, X^{\prime}\right)=\left(\begin{array}{cc}
\frac{\delta \psi_{\alpha}(X)}{\delta J_{\beta}\left(X^{\prime}\right)} & \frac{\delta \psi_{\alpha}(X)}{\delta J_{\beta}^{+}\left(X^{\prime}\right)} \\
\frac{\delta \psi_{\alpha}^{+}(X)}{\delta J_{\beta}\left(X^{\prime}\right)} & \frac{\delta \psi_{\alpha}^{+}(X)}{\delta J_{\beta}^{+}\left(X^{\prime}\right)}
\end{array}\right) \\
& =\left(\begin{array}{cc}
-G_{\alpha \beta}\left(x, \tau ; x^{\prime}, \tau^{\prime}\right) & F_{\alpha \beta}\left(x, \tau ; x^{\prime}, \tau^{\prime}\right) \\
F_{\alpha \beta}^{+}\left(x, \tau ; x^{\prime}, \tau^{\prime}\right) & G_{\beta \alpha}\left(x^{\prime}, \tau^{\prime} ; x, \tau\right)
\end{array}\right),
\end{aligned}
$$

so

$$
\left(\begin{array}{ll}
\frac{\delta J_{\alpha}}{\delta \psi_{\beta}} & \frac{\delta J_{\alpha}}{\delta \psi_{\beta}^{+}} \\
\frac{\delta J_{\alpha}^{+}}{\delta \psi_{\beta}} & \frac{\delta J_{\alpha}^{+}}{\delta \psi_{\beta}^{+}}
\end{array}\right)=\mathcal{G}_{\alpha \beta}^{-1} .
$$

The functional identity is

$$
\begin{aligned}
& \frac{\delta J_{\alpha}(X)}{\delta \psi_{\beta}} \frac{\delta \psi_{\beta}}{\delta J_{\gamma}\left(X^{\prime}\right)}+\frac{\delta J_{\alpha}(X)}{\delta \psi_{\beta}^{+}} \\
& \times \frac{\delta \psi_{\beta}^{+}}{\delta J_{\gamma}\left(X^{\prime}\right)}=\delta_{\alpha \gamma}\left(X-X^{\prime}\right),
\end{aligned}
$$

where the abbreviations

$$
\begin{aligned}
\frac{\delta J_{\alpha}(X)}{\delta \psi_{\beta}} \frac{\delta \psi_{\beta}}{\delta J_{\gamma}\left(X^{\prime}\right)} & \equiv \sum_{\beta} \int \mathrm{d} X^{\prime \prime} \frac{\delta J_{\alpha}(X)}{\delta \psi_{\beta}\left(X^{\prime \prime}\right)} \frac{\delta \psi_{\beta}\left(X^{\prime \prime}\right)}{\delta J_{\gamma}\left(X^{\prime}\right)}, \\
\delta_{\alpha \gamma}\left(X-X^{\prime}\right) & =\delta_{\alpha \gamma} \delta\left(X-X^{\prime}\right)
\end{aligned}
$$

are used. In the Nambu matrix form, it is

$$
\left(\begin{array}{cc}
\frac{\delta J_{\alpha}}{\delta \psi_{\beta}} & \frac{\delta J_{\alpha}}{\delta \psi_{\beta}^{+}} \\
\frac{\delta J_{\alpha}^{+}}{\delta \psi_{\beta}} & \frac{\delta J_{\alpha}^{+}}{\delta \psi_{\beta}^{+}}
\end{array}\right)\left(\begin{array}{ll}
\frac{\delta \psi_{\beta}}{\delta J_{\gamma}} & \frac{\delta \psi_{\beta}}{\delta J_{\gamma}^{+}} \\
\frac{\delta \psi_{\beta}^{+}}{\delta J_{\gamma}} & \frac{\delta \psi_{\beta}^{+}}{\delta J_{\gamma}^{+}}
\end{array}\right)=\left(\begin{array}{cc}
\delta_{\alpha \gamma} & 0 \\
0 & \delta_{\alpha \gamma}
\end{array}\right) \text {. }
$$

The derivatives are

$$
\begin{aligned}
\frac{\delta J_{\alpha}(X)}{\delta \psi_{\beta}\left(X^{\prime}\right)} & =\left(\partial_{\tau} \delta_{\alpha \beta}+K_{\alpha \beta}\right) \delta\left(X-X^{\prime}\right), \\
\frac{\delta J_{\alpha}(X)}{\delta \psi_{\beta}^{+}\left(X^{\prime}\right)} & =g \delta\left(X-X^{\prime}\right)\left\langle\psi_{\alpha}(\tau, \boldsymbol{r}) \psi_{\beta}(\tau, \boldsymbol{r})\right\rangle_{c}, \\
\frac{\delta J_{\alpha}^{+}(X)}{\delta \psi_{\beta}\left(X^{\prime}\right)} & =g \delta\left(X-X^{\prime}\right)\left\langle\psi_{\alpha}^{+}(\tau, \boldsymbol{r}) \psi_{\beta}^{+}(\tau, \boldsymbol{r})\right\rangle, \\
\frac{\delta J_{\alpha}^{+}(X)}{\delta \psi_{\beta}^{+}\left(X^{\prime}\right)} & =\left(\partial_{\tau} \delta_{\alpha \beta}-K_{\beta \alpha}(-\nabla)\right) \delta\left(X-X^{\prime}\right) .
\end{aligned}
$$

For the non-interacting model,

$$
\begin{aligned}
& \mathcal{G}_{0}^{-1}=\left(\begin{array}{cc}
\left(\mathcal{G}_{0}^{-1}\right)_{11} & 0 \\
0 & \left(\mathcal{G}_{0}^{-1}\right)_{22}
\end{array}\right), \\
& \left(\mathcal{G}_{0}^{-1}\right)_{11}=\left(\partial_{\tau} \delta_{\alpha \beta}+K_{\alpha \beta}(\nabla)\right) \delta\left(x-x^{\prime}, \tau-\tau^{\prime}\right),
\end{aligned}
$$




$$
\left(\mathcal{G}_{0}^{-1}\right)_{22}=\left(\partial_{\tau} \delta_{\alpha \beta}-K_{\beta \alpha}(-\nabla)\right) \delta\left(x-x^{\prime}, \tau-\tau^{\prime}\right),
$$

and the gap equation can be cast in the Dyson form:

$$
\begin{aligned}
\mathcal{G}^{-1} & =\mathcal{G}_{0}^{-1}+\left(\begin{array}{cc}
0 & \Sigma_{12} \\
\Sigma_{21} & 0
\end{array}\right), \\
\Sigma_{12} & =g \delta\left(x-x^{\prime}, \tau-\tau^{\prime}\right)\left\langle\psi_{\alpha}(\tau, \boldsymbol{r}) \psi_{\beta}(\tau, \boldsymbol{r})\right\rangle_{c}, \\
\Sigma_{21} & =g \delta\left(x-x^{\prime}, \tau-\tau^{\prime}\right)\left\langle\psi_{\alpha}^{+}(\tau, \boldsymbol{r}) \psi_{\beta}^{+}(\tau, \boldsymbol{r})\right\rangle .
\end{aligned}
$$

\section{B.2 Derivation of energy density expression}

The free energy is

$$
\Omega[\mathcal{G}]=-\frac{1}{\beta}\left\{\frac{1}{2} \operatorname{tr}\left\{-\ln \mathcal{G}+\left[\mathcal{G}_{0}^{-1} \mathcal{G}-1\right]\right\}+\Phi[\mathcal{G}]\right\},
$$

where in the Gaussian approximation,

$$
\Phi[\mathcal{G}]=\frac{g}{2} \int_{\tau, r} F_{\alpha \beta}(X ; X) F_{\beta \alpha}^{+}(X ; X) .
$$

The gap equation can also be obtained using $\frac{\delta}{\delta \mathcal{G}} \Omega[\mathcal{G}]=0$.

The energy difference between the superconducting and normal states can be obtained by differentiating the grand canonical potential with respect to the coupling constant: $\frac{\mathrm{d}}{\mathrm{d} g} \Omega[\mathcal{G}]$. The Green's function $\mathcal{G}$ depends on $g$, but because $\frac{\delta}{\delta \mathcal{G}} \Omega[\mathcal{G}, g]=0$,

$$
\begin{aligned}
\frac{\mathrm{d}}{\mathrm{d} g} \Omega[\mathcal{G}, g] & =\frac{\partial}{\partial g} \Omega[\mathcal{G}, g] \\
& =-\frac{1}{2 \beta} \int_{\tau, r} F_{\alpha \beta}(X ; X) F_{\beta \alpha}^{+}(X ; X) .
\end{aligned}
$$

For the homogeneous state, $F_{\alpha \beta}(X ; X), F_{\beta \alpha}^{+}(X ; X)$ are constant (not dependent on $x, \tau$ ), and we introduce

$$
\begin{aligned}
& F_{\alpha \beta}(X ; X)=\frac{1}{g} \Delta_{\alpha \beta}, \\
& F_{\alpha \beta}^{+}(X ; X)=\frac{1}{g} \Delta_{\alpha \beta}^{+}=\frac{1}{g} \Delta_{\beta \alpha}^{*} .
\end{aligned}
$$

We obtain the free energy formula [66-68]

$$
\begin{aligned}
& \frac{\mathrm{d}}{\mathrm{d} g} \Omega[\mathcal{G}]=-\frac{V}{2} \frac{1}{g^{2}} \operatorname{tr}\left(\Delta \Delta^{+}\right) \rightarrow \\
& \mathrm{d} \Omega[\mathcal{G}]=\frac{V}{2} d\left(\frac{1}{g}\right) \operatorname{tr}\left(\Delta \Delta^{+}\right) .
\end{aligned}
$$

\section{Appendix C. Gap equation and free energy for chiral-symmetry-breaking states}

\section{C.1 Derivation of the gap equation}

We will discuss the nonsuperconducting state with $G_{\alpha \beta}(X, X) \neq n_{c} \delta_{\alpha \beta}, F_{\alpha \beta}\left(X ; X^{\prime}\right)=0$, which appears, for example, in the chiral-symmetry-breaking state. We need only to discuss the equation for $\frac{\delta J_{\alpha}}{\delta \psi_{\beta}}$, as $\frac{\delta J_{\alpha}^{+}}{\delta \psi_{\beta}^{+}}$is just the complex conjugate of $\frac{\delta J_{\alpha}}{\delta \psi_{\beta}}$, and $\frac{\delta J_{\alpha}^{+}}{\delta \psi_{\beta}}=\frac{\delta J_{\alpha}}{\delta \psi_{\beta}^{+}}=0$. We reorganize the equation $\frac{\delta J_{\alpha}}{\delta \psi_{\beta}}$ as

$$
\begin{aligned}
& \frac{\delta J_{\alpha}}{\delta \psi_{\beta}}=\left(\partial_{\tau} \delta_{\alpha \beta}+\widehat{K}_{\alpha \beta}\right) \delta\left(X-X^{\prime}\right)-g \delta\left(X-X^{\prime}\right) \\
& \quad \times\left\{\left\langle\psi_{\alpha}(X) \psi_{\beta}^{+}(X)\right\rangle_{c}-\frac{1}{4} \delta_{\alpha \beta}\right. \\
& \left.\quad \times \sum\left\langle\psi_{\gamma}(X) \psi_{\gamma}^{+}(X)\right\rangle_{c}\right\}+g \delta_{\alpha \beta} \delta\left(X-X^{\prime}\right) \\
& \quad \times\left\{\left\langle\psi_{\beta}(X) \psi_{\beta}^{+}(X)\right\rangle_{c}-\sum\left\langle\psi_{\gamma}(X) \psi_{\gamma}^{+}(X)\right\rangle_{c}\right\} .
\end{aligned}
$$

The last two terms are proportional to $\delta_{\alpha \beta}$ and can be absorbed into the chemical potential:

$$
\begin{gathered}
\mu+g \delta_{\alpha \beta}\left\{\sum\left\langle\psi_{\gamma}(X) \psi_{\gamma}^{+}(X)\right\rangle_{c}\right. \\
\left.-\left\langle\psi_{\beta}(X) \psi_{\beta}^{+}(X)\right\rangle_{c}\right\}=\mu_{R} .
\end{gathered}
$$

The gap equation can be recast as

$$
\begin{aligned}
& G^{-1}=G_{0}^{-1}+g \delta\left(X-X^{\prime}\right) G^{\prime}\left(X ; X^{\prime}\right), \\
& G_{0}^{-1}=\left(\partial_{\tau} \delta_{\alpha \beta}+\widehat{K}_{\alpha \beta}\right) \delta \delta\left(X-X^{\prime}\right), \\
& G_{\alpha \beta}^{\prime}\left(X ; X^{\prime}\right)=-\left\{\left\langle\psi_{\alpha}(X) \psi_{\beta}^{+}\left(X^{\prime}\right)\right\rangle_{c}\right. \\
& \left.\quad-\frac{1}{4} \delta_{\alpha \beta} \sum\left\langle\psi_{\gamma}(X) \psi_{\gamma}^{+}\left(X^{\prime}\right)\right\rangle_{c}\right\},
\end{aligned}
$$

where $G_{\alpha \beta}^{\prime}$ is the traceless part of $G_{\alpha \beta}$, and the chemical potential in $\widehat{K}_{\alpha \beta}$ is $\mu_{R}$. The free energy is now

$$
\Omega[\mathcal{G}]=-(\beta)^{-1}\left\{\operatorname{tr}\left\{-\ln G+\left[G_{0}^{-1} G-1\right]\right\}+\Phi[G]\right\},
$$

where

$$
\Phi[\mathcal{G}]=-\frac{g}{2} \int_{\tau, r} G_{\alpha \beta}^{\prime}(x, \tau ; x, \tau) G_{\beta \alpha}^{\prime}(x, \tau ; x, \tau) .
$$

A similar free energy equation can be obtained:

$$
\begin{aligned}
& \frac{\mathrm{d}}{\mathrm{d} g} \Omega[\mathcal{G}, g]=\frac{\partial}{\partial g} \Omega[\mathcal{G}, g] \\
& \quad=\frac{1}{2 \beta} \int_{\tau, r} G_{\alpha \beta}^{\prime}(x, \tau ; x, \tau) G_{\beta \alpha}^{\prime}(x, \tau ; x, \tau) .
\end{aligned}
$$

C.2 Details of the condensate and energy calculation

By using the integral

$$
\begin{aligned}
& \int_{\omega} \frac{1}{m^{2}+\left(\hbar v_{F} k\right)^{2}-(\mu+\mathrm{i} \omega)^{2}} \\
& =\frac{\pi}{\sqrt{m^{2}+\left(\hbar v_{F} k\right)^{2}}} \Theta\left(\sqrt{m^{2}+\left(\hbar v_{F} k\right)^{2}}-\mu\right),
\end{aligned}
$$

and

Dingping Li, et al., Front. Phys. 10, 107402 (2015) 


$$
\begin{aligned}
& \frac{1}{(2 \pi)^{3}} \int_{\omega, p} \frac{1}{m^{2}+p^{2}-(\mu+\mathrm{i} \omega)^{2}} \\
& =\frac{1}{4 \pi} \int_{p=0}^{\Lambda} \frac{p \Theta\left(\sqrt{m^{2}+p^{2}}-\mu\right)}{\sqrt{m^{2}+p^{2}}} \\
& =\left\{\begin{array}{l}
\frac{1}{4 \pi}\left(\sqrt{m^{2}+\Lambda^{2}}-m\right), \mu \leqslant m \\
\frac{1}{4 \pi}\left(\sqrt{m^{2}+\Lambda^{2}}-\mu\right), \mu>m
\end{array}\right.
\end{aligned}
$$

the gap equation is obtained as

$$
m=-\frac{g}{4 \pi\left(\hbar v_{F}\right)^{2}} \begin{cases}\left(\sqrt{m^{2}+\Lambda^{2}}-m\right), & \mu \leqslant m ; \\ \left(\sqrt{m^{2}+\Lambda^{2}}-\mu\right), & \mu>m .\end{cases}
$$

\section{Appendix D. Symmetries of the Dirac model: Chiral nonrelativistic $S U(2)$}

The charge algebra is generally $\left[Q, Q_{i}\right]=0 ;\left[Q_{i}, Q_{j}\right]=$ $i \varepsilon_{i j k} Q_{k}$. Indeed, all three chiral charges commute with the density

$$
\begin{aligned}
{\left[\rho(x), Q_{i}\right] } & =\int_{y}\left[\psi^{\dagger}(x) \psi(x), \psi^{\dagger}(y) T_{i} \psi(y)\right] \\
& =\psi^{\dagger}(x)\left[I, T_{i}\right] \psi(x)=0
\end{aligned}
$$

and the kinetic term

$$
\begin{aligned}
{\left[H, Q_{i}\right] } & =\int_{y}\left[\psi^{\dagger}(x)\left(\alpha_{1} p_{1}+\alpha_{2} p_{2}\right) \psi(x), \psi^{\dagger}(y) T_{i} \psi(y)\right] \\
& =\psi^{\dagger}(x)\left[\alpha_{1} p_{1}+\alpha_{2} p_{2}, T_{i}\right] \psi(x)=0 .
\end{aligned}
$$

The three generator matrices [Eq. (27)] constituting $S U(2),\left[T_{i}, T_{j}\right]=\mathrm{i} \varepsilon_{i j k} T_{k}$, commute with both $\gamma_{0} \gamma_{1}$ and $\gamma_{0} \gamma_{2}$, which appear in the noninteracting Hamiltonian [Eq. (1)]. The density-density interaction component of the Hamiltonian also commutes with the $S U(2)$ charges because

$$
\begin{aligned}
{\left[\rho(r), Q_{i}\right] } & =\int_{r^{\prime}}\left[\psi^{\dagger}(r) \psi(r), \psi^{\dagger}\left(r^{\prime}\right) T_{i} \psi\left(r^{\prime}\right)\right] \\
& =\psi^{\dagger}(r)\left[I, T_{i}\right] \psi(r)=0 .
\end{aligned}
$$

Correspondingly, the action in Eq. (22) is invariant under $\delta \psi=\mathrm{i} T_{i} \psi ; \delta \bar{\psi}=\mathrm{i} \bar{\psi} \gamma_{0} T_{i} \gamma_{0}$. Indeed, both the energy term,

$$
\begin{aligned}
\delta A_{0} & =-\int\left[\mathrm{i} \bar{\psi} \gamma_{0} T_{i} \gamma_{0} \gamma_{0} \partial_{0} \psi+\bar{\psi}^{s} \gamma_{0} \partial_{0} \mathrm{i} T_{i} \psi\right] \\
& =-\int\left[-\mathrm{i} \bar{\psi} \gamma_{0} T_{i} \partial_{0} \psi^{s}+\bar{\psi}^{s} \gamma_{0} \partial_{0} \mathrm{i} T_{i} \psi^{s}\right]=0,
\end{aligned}
$$

and the momentum terms,

$$
\begin{aligned}
\delta A_{1} & =-\int\left[\mathrm{i} \bar{\psi} \gamma_{0} T_{i} \gamma_{0}(\gamma \cdot \partial) \psi^{s}+\bar{\psi}(\gamma \cdot \partial) \mathrm{i} T_{i} \psi\right] \\
& =-\mathrm{i} \int \bar{\psi}\left[\gamma_{0} T_{i} \gamma_{0} \gamma+\gamma T_{i}\right] \cdot \partial \psi=0,
\end{aligned}
$$

are invariant. The nonrelativistic interaction term is

$$
\begin{aligned}
\delta A & =v \int\left[\mathrm{i} \bar{\psi} \gamma_{0} T_{i} \gamma_{0} \gamma_{0} \psi+\bar{\psi} \gamma_{0} \mathrm{i} T_{i} \psi\right]\left(\bar{\psi}^{r} \gamma_{0} \psi^{r}\right) \\
& =\mathrm{i} v \int\left[-\bar{\psi} \gamma_{0} T_{i} \psi+\bar{\psi} \gamma_{0} T_{i} \psi\right]\left(\bar{\psi}^{r} \gamma_{0} \psi^{r}\right)=0 .
\end{aligned}
$$

\section{References}

1. S. Q. Shen, Topological Insulators, Heidelberg: SpringerVerlag, 2012

2. X. L. Qi and S. C. Zhang, Topological insulators and superconductors, Rev. Mod. Phys. 83(4), 1057 (2011)

3. M. Z. Hasan and C. L. Kane, Colloquium: Topological insulators, Rev. Mod. Phys. 82(4), 3045 (2010)

4. M. I. Katsnelson, Graphene: Carbon in Two Dimensions, Cambridge: Cambridge University Press, 2012

5. A. H. Castro Neto, F. Guinea, N. M. R. Peres, K. S. Novoselov, and A. K. Geim, The electronic properties of graphene, Rev. Mod. Phys. 81(1), 109 (2009)

6. A. M. Black-Schaffer and S. Doniach, Resonating valence bonds and mean-field d-wave superconductivity in graphite, Phys. Rev. B 75(13), 134512 (2007)

7. S. Pathak, V. B. Shenoy, and G. Baskaran, Possible hightemperature superconducting state with a d+id pairing symmetry in doped graphene, Phys. Rev. B 81(8), 085431 (2010)

8. R. Nandkishore, L. S. Levitov, and A. V. Chubukov, Chiral superconductivity from repulsive interactions in doped graphene, Nat. Phys. 8(2), 158 (2012)

9. B. Roy and I. F. Herbut, Unconventional superconductivity on honeycomb lattice: Theory of Kekule order parameter, Phys. Rev. B 82(3), 035429 (2010)

10. D. V. Khveshchenko, Ghost excitonic insulator transition in layered graphite, Phys. Rev. Lett. 87(24), 246802 (2001)

11. O. V. Gamayun, E. V. Gorbar, and V. P. Gusynin, Gap generation and semimetal-insulator phase transition in graphene, Phys. Rev. B 81(7), 075429 (2010)

12. B. Rosenstein and B. J. Warr, Dynamical symmetry breaking in 2+1 dimensions, Phys. Lett. B 218(4), 465 (1989)

13. M. V. Ulybyshev, P. V. Buividovich, M. I. Katsnelson, and M. I. Polikarpov, Monte Carlo study of the semimetalinsulator phase transition in monolayer graphene with a realistic interelectron interaction potential, Phys. Rev. Lett. 111(5), 056801 (2013)

14. J. Martin, B. E. Feldman, R. T. Weitz, M. T. Allen, and A. Yacoby, Local compressibility measurements of correlated states in suspended bilayer graphene, Phys. Rev. Lett. 105(25), 256806 (2010)

15. R. T. Weitz, M. T. Allen, B. E. Feldman, J. Martin, and A. Yacoby, Broken-symmetry states in doubly gated suspended bilayer graphene, Science 330(6005), 812 (2010)

16. F. Freitag, J. Trbovic, M. Weiss, and C. Schonenberger, 
Spontaneously gapped ground state in suspended bilayer graphene, Phys. Rev. Lett. 108(7), 076602 (2012)

17. L. Velasco, W. Jing, Y. Bao, P. Lee, V. Kratz, M. Aji, C. N. Bockrath, C. Lau, R. Varma, D. Stillwell, F. Smirnov, J. Zhang, J. Jung, and A. H. MacDonald, Transport spectroscopy of symmetry-broken insulating states in bilayer graphene, Nat. Nanotechnol. 7(3), 156 (2012)

18. V. M. Nabutovskii and B. Ya. Shapiro, Superconductivity in a system of interacting localized and delocalized electrons, Zh. Eksp. Teor. Fiz. 84, 422 (1983) [Sov. Phys. JETP 57(1), 245 (1983)]

19. P. A. Lee, N. Nagaosa, and X. G. Wen, Doping a Mott insulator: Physics of high-temperature superconductivity, Rev. Mod. Phys. 78(1), 17 (2006)

20. J. Orenstein and A. J. Millis, Advances in the physics of high-temperature superconductivity, Science 288(5465), 468 (2000)

21. J. Singleton and C. Mielke, Quasi-two-dimensional organic superconductors: A review, Contemp. Phys. 43(2), 63 (2002)

22. I. N. Khlyustikov and A. I. Buzdin, Twinning-plane superconductivity, Adv. Phys. 36(3), 271 (1987)

23. X. Zhu, L. Santos, R. Sankar, S. Chikara, C. Howard, F. C. Chou, C. Chamon, and M. El-Batanouny, Interaction of phonons and Dirac fermions on the surface of $\mathrm{Bi}_{2} \mathrm{Se}_{3}$ : A strong Kohn anomaly, Phys. Rev. Lett. 107(18), 186102 (2011)

24. C. W. Luo, H. J. Wang, S. A. Ku, H. J. Chen, T. T. Yeh, J. Y. Lin, K. H. Wu, J. Y. Juang, B. L. Young, T. Kobayashi, C. M. Cheng, C. H. Chen, K. D. Tsuei, R. Sankar, F. C. Chou, K. A. Kokh, O. E. Tereshchenko, E. V. Chulkov, Yu. M. Andreev, and G. D. Gu, Snapshots of Dirac fermions near the Dirac point in topological insulators, Nano Lett. 13(12), 5797 (2013)

25. X. Zhu, L. Santos, C. Howard, R. Sankar, F. C. Chou, C. Chamon, and M. El-Batanouny, Electron-phonon coupling on the surface of the topological insulator $\mathrm{Bi}_{2} \mathrm{Se}_{3}$ determined from surface-phonon dispersion measurements, Phys. Rev. Lett. 108(18), 185501 (2012)

26. S. Das Sarma and Q. Z. Li, Many-body effects and possible superconductivity in the two-dimensional metallic surface states of three-dimensional topological insulators, Phys. Rev. B 88, 081404(R) (2013)

27. Z. H. Pan, A. V. Fedorov, D. Gardner, Y. S. Lee, S. Chu, and T. Valla, Measurement of an exceptionally weak electronphonon coupling on the surface of the topological insulator $\mathrm{Bi}_{2} \mathrm{Se}_{3}$ using angle-resolved photoemission spectroscopy, Phys. Rev. Lett. 108(18), 187001 (2012)

28. V. Parente, A. Tagliacozzo, F. von Oppen, and F. Guinea, Electron-phonon interaction on the surface of a threedimensional topological insulator, Phys. Rev. B 88(7), 075432 (2013)

29. M. Cheng, R. M. Lutchyn, and S. Das Sarma, Topological protection of Majorana qubits, Phys. Rev. B 85(16), 165124
(2012)

30. D. Li, B. Rosenstein, B. Ya. Shapiro, and I. Shapiro, Quantum critical point in the superconducting transition on the surface of a topological insulator, Phys. Rev. B 90(5), 054517 (2014)

31. H. Zhang, C. X. Liu, X. L. Qi, X. Dai, Z. Fang, and S. C. Zhang, Topological insulators in $\mathrm{Bi}_{2} \mathrm{Se}_{3}, \mathrm{Bi}_{2} \mathrm{Te}_{3}$ and $\mathrm{Sb}_{2} \mathrm{Te}_{3}$ with a single Dirac cone on the surface, Nat. Phys. 5(6), 438 (2009)

32. J. G. Checkelsky, Y. S. Hor, R. J. Cava, and N. P. Ong, Bulk band gap and surface state conduction observed in voltagetuned crystals of the topological insulator $\mathrm{Bi}_{2} \mathrm{Se}_{3}$, Phys. Rev. Lett. 106(19), 196801 (2011)

33. D. Kim, S. Cho, N. P. Butch, P. Syers, K. Kirshenbaum, S. Adam, J. Paglione, and M. S. Fuhrer, Surface conduction of topological Dirac electrons in bulk insulating $\mathrm{Bi}_{2} \mathrm{Se}_{3}$, Nat. Phys. 8(6), 459 (2012)

34. C. K. Lu and I. F. Herbut, Pairing symmetry and vortex zero mode for superconducting Dirac fermions, Phys. Rev. B 82(14), 144505 (2010)

35. M. Sato and S. Fujimoto, Topological phases of noncentrosymmetric superconductors: Edge states, Majorana fermions, and non-Abelian statistics, Phys. Rev. B 79(9), 094504 (2009)

36. S. Sachdev, Quantum Phase Transitions, Cambridge: Cambridge University Press, 2011

37. I. Herbut, A Modern Approach to Critical Phenomena, Cambridge: Cambridge University Press, 2010

38. D. J. Amit, Field Theory, The Renormalization Group and Critical Phenomena, London: World Scientific, 2005

39. B. Rosenstein, B. J. Warr, and S. H. Park, Four-fermion theory is renormalizable in $2+1$ dimensions, Phys. Rev. Lett. 62(13), 1433 (1989)

40. B. Rosenstein, B. J. Warr, and S. H. Park, Dynamical symmetry breaking in four-fermion interaction models, Phys. Rep. 205(2), 59 (1991)

41. G. Gat, A. Kovner, and B. Rosenstein, Chiral phase transitions in $d=3$ and renormalizability of four-Fermi interactions, Nucl. Phys. B 385(1-2), 76 (1992)

42. B. Rosenstein, Hoi-Lai Yu, and A. Kovner, Critical exponents of new universality classes, Phys. Lett. B 314(3-4), 381 (1993)

43. R. Schneider, A. G. Zaitsev, D. Fuchs, and H. v. Löhneysen, Superconductor-insulator quantum phase transition in disordered FeSe thin films, Phys. Rev. Lett. 108(25), 257003 (2012)

44. V. N. Kotov, B. Uchoa, V. M. Pereira, F. Guinea, and A. H. Castro Neto, Electron-electron interactions in graphene: Current status and perspectives, Rev. Mod. Phys. 84(3), 1067 (2012)

45. H. A. Fertig, Energy spectrum of a layered system in a strong magnetic field, Phys. Rev. B 40(2), 1087 (1989) 
46. S. Q. Murphy, J. P. Eisenstein, G. S. Boebinger, L. N. Pfeiffer, and K. W. West, Many-body integer quantum Hall effect: Evidence for new phase transitions, Phys. Rev. Lett. 72(5), 728 (1994)

47. I. B. Spielman, J. P. Eisenstein, L. N. Pfeiffer, and K. W. West, Resonantly enhanced tunneling in a double layer quantum Hall ferromagnet, Phys. Rev. Lett. 84(25), 5808 (2000)

48. I. B. Spielman, J. P. Eisenstein, L. N. Pfeiffer, and K. W. West, Observation of a linearly dispersing collective mode in a quantum Hall ferromagnet, Phys. Rev. Lett. 87(3), 036803 (2001)

49. Y. Yoon, L. Tiemann, S. Schmult, W. Dietsche, K. von Klitzing, and W. Wegscheider, Interlayer tunneling in counterflow experiments on the excitonic condensate in quantum Hall bilayers, Phys. Rev. Lett. 104(11), 116802 (2010)

50. A. D. K. Finck, J. P. Eisenstein, L. N. Pfeiffer, and K. W. West, Exciton transport and Andreev reflection in a bilayer quantum Hall system, Phys. Rev. Lett. 106(23), 236807 (2011)

51. X. Huang, W. Dietsche, M. Hauser, and K. von Klitzing, Coupling of Josephson currents in quantum Hall bilayers, Phys. Rev. Lett. 109(15), 156802 (2012)

52. B. Seradjeh, J. E. Moore, and M. Franz, Exciton condensation and charge fractionalization in a topological insulator film, Phys. Rev. Lett. 103(6), 066402 (2009)

53. Z. Wang, N. Hao, Z. G. Fu, and P. Zhang, Excitonic condensation for the surface states of topological insulator bilayers, New J. Phys. 14(6), 063010 (2012)

54. D. K. Efimkin, Yu. E. Lozovik, and A. A. Sokolik, Electronhole pairing in a topological insulator thin film, Phys. Rev. B 86(11), 115436 (2012)

55. S. Rist, A. A. Varlamov, A. H. MacDonald, R. Fazio, and M. Polini, Photoemission spectra of massless Dirac fermions on the verge of exciton condensation, Phys. Rev. B 87(7), 075418 (2013)

56. D. W. Zhang, Z. D. Wang, and S. L. Zhu, Relativistic quantum effects of Dirac particles simulated by ultracold atoms, Front. Phys. 7(1), 31 (2012)

57. L. Fu and E. Berg, Odd-parity topological superconductors: Theory and application to $\mathrm{Cu}_{x} \mathrm{Bi}_{2} \mathrm{Se}_{3}$, Phys. Rev. Lett. 105(9), 097001 (2010)

58. B. Rosenstein, B. Ya. Shapiro, D. Li, and I. Shapiro, Triplet superconductivity in 3D Dirac semi-metal due to exchange interaction, J. Phys.: Condens. Matter 27(2), 025701 (2015)

59. A. A. Abrikosov, L. P. Gor'kov, and I. E. Dzyaloshinskii, Quantum Field Theoretical Methods in Statistical Physics, New York: Pergamon Press, 1965

60. E. M. Lifshits and L. P. Pitaeskii, Course of Theoretical Physics (Vol. 9): Statistical Physics, Part 2, Oxford: Prgamon Press, 1980

61. J. M. Cornwall, R. Jackiw, and E. Tomboulis, Effective action for composite operators, Phys. Rev. D 10(8), 2428 (1974)
62. R. Haussmann, Self-Consistent Quantum-Field Theory and Bosonization for Strongly Correlated Electron Systems, Springer, 1999

63. Z. J. Wang, Y. Sun, X. Q. Chen, C. Franchini, G. Xu, H. M. Weng, X. Dai, and Z. Fang, Dirac semimetal and topological phase transitions in $\mathrm{A}_{3} \mathrm{Bi}(\mathrm{A}=\mathrm{Na}, \mathrm{K}, \mathrm{Rb})$, Phys. Rev. $B$ 85(19), 195320 (2012)

64. P. Hosur, X. Dai, Z. Fang, and X. L. Qi, Time-reversalinvariant topological superconductivity in doped Weyl semimetals, Phys. Rev. B 90(4), 045130 (2014)

65. V. P. Gusynin, S. G. Sharapov, and J. P. Carbotte, Ac conductivity of graphene: From tight-binding model to $2+1$ dimensional quantum electrodynamics, Int. J. Mod. Phys. B 21(27), 4611 (2007)

66. A. A. Abrikosov, On the magnetic properties of superconductors of the second group, Zh. Eksp. Teor. Fiz. 32, 1442 (1957) [Sov. Phys. JETP 5(6), 1174 (1957)]

67. J. D. Ketterson and S. N. Song, Superconductivity, Cambridge: Cambridge University Press, 1999

68. B. Rosenstein and D. Li, Ginzburg-Landau theory of type II superconductors in magnetic field, Rev. Mod. Phys. 82(1), 109 (2010)

69. I. F. Herbut, V. Juricic, and O. Vafek, Relativistic Mott criticality in graphene, Phys. Rev. B 80(7), 075432 (2009)

70. L. Janssen and I. F. Herbut, Antiferromagnetic critical point on graphene's honeycomb lattice: A functional renormalization group approach, Phys. Rev. B 89(20), 205403 (2014)

71. L. Del Debbio, S. J. Hands, and J. C. Mehegan, Threedimensional thirring model for small Nf, Nucl. Phys. B 502(1-2), 269 (1997)

72. I. M. Barbour, N. Psycharis, E. Focht, W. Franzki, and J. Jersak, Strongly coupled lattice gauge theory with dynamical fermion mass generation in three dimensions, Phys. Rev. D 58(7), 074507 (1998)

73. S. Chandrasekharan and A. Li, Fermion bag solutions to some sign problems in four-fermion field theories, Phys. Rev. D 85(9), 091502 (2012)

74. S. Chandrasekharan, Solutions to sign problems in lattice Yukawa models, Phys. Rev. D 86(2), 021701 (2012)

75. S. Chandrasekharan and Anyi Li, Quantum critical behavior in three dimensional lattice Gross-Neveu models, Phys. Rev. D 88, 021701(R) (2013)

76. F. F. Assaad and I. F. Herbut, Pinning the order: The nature of quantum criticality in the Hubbard model on honeycomb lattice, Phys. Rev. X 3, 031010 (2013)

77. S. Sorella, Y. Otsuka, and S. Yunoki, Absence of a spin liquid phase in the Hubbard model on the honeycomb lattice, Scientific Reports 2, 992 (2012)

78. B. W. Lee, Chiral Dynamics, New York: Gordon and Breach, 1972

79. Z. H. Pan, A. V. Fedorov, D. Gardner, Y. S. Lee, S. Chu, and T. Valla, Measurement of an exceptionally weak electronphonon coupling on the surface of the topological insula- 
tor $\mathrm{Bi}_{2} \mathrm{Se}_{3}$ using angle-resolved photoemission spectroscopy, Phys. Rev. Lett. 108(18), 187001 (2012)

80. V. Parente, A. Tagliacozzo, F. von Oppen, and F. Guinea, Electron-phonon interaction on the surface of a threedimensional topological insulator, Phys. Rev. B 88(7), 075432 (2013)

81. Y. S. Hor, A. J. Williams, J. G. Checkelsky, P. Roushan, J. Seo, Q. Xu, H. W. Zandbergen, A. Yazdani, N. P. Ong, and R. J. Cava, Superconductivity in $\mathrm{Cu}_{x} \mathrm{Bi}_{2} \mathrm{Se}_{3}$ and its implications for pairing in the undoped topological insulator, Phys. Rev. Lett. 104(5), 057001 (2010)

82. L. A. Wray, S. Y. Xu, Y. Xia, Y. S. Hor, D. Qian, A. V. Fedorov, H. Lin, A. Bansil, R. J. Cava, and M. Z. Hasan, Observation of topological order in a superconducting doped topological insulator, Nat. Phys. 6(11), 855 (2010)

83. G. Koren, T. Kirzhner, E. Lahoud, K. Chashka, and A. Kanigel, Proximity-induced superconductivity in topological $\mathrm{Bi}_{2} \mathrm{Te}_{2} \mathrm{Se}$ and $\mathrm{Bi}_{2} \mathrm{Se}_{3}$ films: Robust zero-energy bound state possibly due to Majorana fermions, Phys. Rev. B 84(22), 224521 (2011)

84. P. H. Le, W.-Y. Tzeng, H.-J. Chen, C. W. Luo, J.Y. Lin, and J. Leu, Superconductivity in textured Bi clusters $/ \mathrm{Bi}_{2} \mathrm{Te}_{3}$ films, APL Mat. 2, 096105 (2014)

85. K. Kirshenbaum, P. S. Syers, A. P. Hope, N. P. Butch, J. R. Jeffries, S. T. Weir, J. J. Hamlin, M. B. Maple, Y. K. Vohra, and J. Paglione, Pressure-induced unconventional superconducting phase in the topological insulator $\mathrm{Bi}_{2} \mathrm{Se}_{3}$, Phys. Rev. Lett. 111(8), 087001 (2013)

86. Z. K. Liu, B. Zhou, Y. Zhang, Z. J. Wang, H. M. Weng, D. Prabhakaran, S. K. Mo, Z. X. Shen, Z. Fang, X. Dai, Z. Hussain, and Y. L. Chen, Discovery of a three-dimensional topological Dirac semimetal, $\mathrm{Na}_{3} \mathrm{Bi}$, Science 343(6173), 864 (2014)

87. S. Y. Xu, C. Liu, S. K. Kushwaha, T. R. Chang, J. W. Krizan, R. Sankar, C. M. Polley, J. Adell, T. Balasubramanian, K. Miyamoto, N. Alidoust, G. Bian, M. Neupane, I. Belopolski, H. T. Jeng, C. Y. Huang, W. F. Tsai, H. Lin, F. C. Chou, T. Okuda, A. Bansil, R. J. Cava, and M. Z. Hasan, Observation of a bulk 3D Dirac multiplet, Lifshitz transition, and nestled spin states in $\mathrm{Na}_{3} \mathrm{Bi}$, arXiv: 1312.7624 (2013)

88. M. Orlita, D. M. Basko, M. S. Zholudev, F. Teppe, W.
Knap, V. I. Gavrilenko, N. N. Mikhailov, S. A. Dvoretskii, P. Neugebauer, C. Faugeras, A. L. Barra, G. Martinez, and M. Potemski, Observation of three-dimensional massless Kane fermions in a zinc-blende crystal, Nat. Phys. 10(3), 233 (2014)

89. G. Xu, H. Weng, Z. Wang, X. Dai, and Z. Fang, Chern semimetal and the quantized anomalous Hall effect in $\mathrm{HgCr}_{2} \mathrm{Se}_{4}$, Phys. Rev. Lett. 107(18), 186806 (2011)

90. Z. J. Wang, H. M. Weng, Q. Wu, X. Dai, and Z. Fang, Three-dimensional Dirac semimetal and quantum transport in $\mathrm{Cd}_{3} \mathrm{As}_{2}$, Phys. Rev. B 88(12), 125427 (2013)

91. M. Neupane, S. Y. Xu, N. Alidoust, G. Bian, C. Liu, I. Belopolski, T. R. Chang, H. T. Jeng, H. Lin, A. Bansil, F. C. Chou, and M. Z. Hasan, Observation of quantumtunneling modulated spin texture in ultrathin topological insulator $\mathrm{Bi}_{2} \mathrm{Se}_{3}$ films, Nat. Commun. 05, 3786 (2014), arXiv: $1404.2830 \mathrm{v} 1$

92. Y. Fuseya, M. Ogata, and H. Fukuyama, Interband contributions from the magnetic field on Hall effects for Dirac electrons in bismuth, Phys. Rev. Lett. 102(6), 066601 (2009)

93. P. Hosur, S. A. Parameswaran, and A. Vishwanath, Charge transport in Weyl semimetals, Phys. Rev. Lett. 108(4), 046602 (2012)

94. T. Kariyado and M. Ogata, Three-dimensional Dirac electrons at the Fermi energy in cubic inverse perovskites: $\mathrm{Ca}_{3} \mathrm{PbO}$ and its family, J. Phys. Soc. Jpn. 80(8), 083704 (2011)

95. T. Kariyado and M. Ogata, Low-energy effective hamiltonian and the surface states of $\mathrm{Ca}_{3} \mathrm{PbO}$, J. Phys. Soc. Jpn. 81(6), 064701 (2012)

96. P. Delplace, J. Li, and D. Carpentier, Topological Weyl semi-metal from a lattice model, EPL (Europhysics Letters) 97(6), 67004 (2012)

97. B. Rosenstein and M. Lewkowicz, Dynamics of electric transport in interacting Weyl semimetals, Phys. Rev. B 88(4), 045108 (2013)

98. M. N. Ali, Q. D. Gibson, T. Klimczuk, and R. J. Cava, Noncentrosymmetric superconductor with a bulk threedimensional Dirac cone gapped by strong spin-orbit coupling, Phys. Rev. B 89(2), 020505 (2014) (R) 